\title{
Search for physics beyond the standard model in events with two leptons, jets, and missing transverse momentum in pp collisions at $\sqrt{s}=8 \mathrm{TeV}$
}

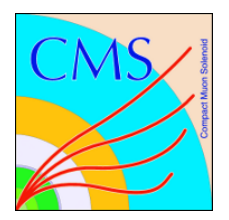

\section{The CMS collaboration}

E-mail: cms-publication-committee-chair@cern.ch

ABSTRACT: A search is presented for physics beyond the standard model in final states with two opposite-sign same-flavor leptons, jets, and missing transverse momentum. The data sample corresponds to an integrated luminosity of $19.4 \mathrm{fb}^{-1}$ of proton-proton collisions at $\sqrt{s}=8 \mathrm{TeV}$ collected with the CMS detector at the CERN LHC in 2012. The analysis focuses on searches for a kinematic edge in the invariant mass distribution of the oppositesign same-flavor lepton pair and for final states with an on-shell Z boson. The observations are consistent with expectations from standard model processes and are interpreted in terms of upper limits on the production of supersymmetric particles.

KEYwords: Supersymmetry, Hadron-Hadron Scattering

ARXiv EPrint: 1502.06031 


\section{Contents}

1 Introduction 1

2 Detector and trigger $\quad 2$

3 Signal scenarios 3

4 Event selection, reconstruction, and search strategy 4

$\begin{array}{lll}5 & \text { Background estimates } & 7\end{array}$

$\begin{array}{lll}5.1 & \text { Flavor-symmetric backgrounds } & 7\end{array}$

5.2 SM Drell-Yan background 9

$\begin{array}{llr}6 & \text { Kinematic fit } & 10\end{array}$

$\begin{array}{llr}7 & \text { Results } & 11\end{array}$

8 Uncertainties in signal modeling $\quad 16$

$\begin{array}{lll}9 & \text { Interpretation } & 17\end{array}$

$\begin{array}{ll}10 \text { Summary } & 18\end{array}$

$\begin{array}{ll}\text { The CMS collaboration } & 23\end{array}$

\section{Introduction}

This paper presents a search for physics beyond the standard model (SM) in events containing a pair of opposite-sign same-flavor (SF) electrons or muons, jets, and an imbalance in transverse momentum. The analysis is based on a sample of proton-proton (pp) collisions collected at a center-of-mass energy of $8 \mathrm{TeV}$ with the CMS detector [1] at the CERN LHC in 2012 and corresponds to an integrated luminosity of $19.4 \mathrm{fb}^{-1}$.

The invariant mass distribution of the two-lepton system can exhibit an excess that increases with the dilepton mass, followed by a sharp decrease and thus an "edge", if the two leptons originate from the decay of an on-shell heavy neutral particle. This kind of signature is fairly generic for models of physics beyond the SM (BSM), assumes an isotropic decay, and is purely kinematic in origin. In models of supersymmetry (SUSY) [2], an edge with a triangular shape is expected in the cascade process $\widetilde{\chi}_{2}^{0} \rightarrow \ell \widetilde{\ell} \rightarrow \widetilde{\chi}_{1}^{0} \ell^{+} \ell^{-}[3]$, where $\tilde{\chi}_{2}^{0}$ and $\tilde{\chi}_{1}^{0}$ are respectively the next-to-lightest and lightest neutralino, with $\tilde{\ell}$ a selectron or smuon, the SUSY partners of an electron or muon. Alternatively, the $\widetilde{\chi}_{2}^{0}$ can undergo a three-body decay to $\widetilde{\chi}_{1}^{0} \ell^{+} \ell^{-}$through a virtual $\mathrm{Z}^{*}$ boson, also yielding an edge in the dilepton 
mass spectrum but with a more rounded shape. Another possibility is the decay of a $\widetilde{\chi}_{2}^{0}$ to an on-shell $\mathrm{Z}$ boson, $\tilde{\chi}_{2}^{0} \rightarrow \widetilde{\chi}_{1}^{0} \mathrm{Z}$. This latter process does not produce an edge but rather a dilepton mass peak near $91 \mathrm{GeV}$. These processes arise as a consequence of the gaugecoupling structure of SUSY and are a characteristic feature of SUSY decay chains. Their relative importance depends on the SUSY mass hierarchy and is thus model dependent.

This search is therefore motivated by the possible existence of the fairly generic signal shape of an edge, or of a peak at the $\mathrm{Z}$ boson mass, that would be visible in the invariant mass distribution of the two leptons. The position of the edge would give an indication of the unknown BSM mass hierarchy. The dilepton invariant mass provides a search variable that is unaffected by uncertainties in the jet energy scale and resolution, and the search for an edge is therefore complementary to searches based solely on hadronic quantities.

The CMS Collaboration previously presented two searches for BSM physics based on events with an opposite-sign SF lepton pair: a search for an edge in the dilepton mass spectrum outside the $\mathrm{Z}$ boson mass region [4], and a search for events containing onshell $\mathrm{Z}$ bosons [5]. Both these studies were conducted using the $7 \mathrm{TeV}$ CMS data sample collected in 2011. The present study updates and combines these two analyses, using the $8 \mathrm{TeV}$ data sample. Searches for SUSY in opposite-sign dilepton final states, but targeting different production and/or decay mechanisms, are presented by the ATLAS Collaboration in refs. [6, 7].

A brief description of the CMS detector is given in section 2. Signal models studied in this analysis are described in section 3. Section 4 outlines the event selection and simulation. The background estimation methods are presented in section 5. A fitting procedure used to search for an edge is described in section 6. The results of the search are presented in section 7 . Systematic uncertainties associated with the signal modeling are discussed in section 8 and the results of the search are interpreted in the context of the signal models in section 9. A summary is presented in section 10.

\section{Detector and trigger}

The central feature of the CMS detector is a superconducting solenoid of $6 \mathrm{~m}$ internal diameter that produces an axial magnetic field of $3.8 \mathrm{~T}$. A silicon pixel and strip tracker, a lead tungstate crystal electromagnetic calorimeter, and a brass/plastic-scintillator hadron calorimeter are positioned within the field volume. Iron and quartz-fiber hadron calorimeters are located outside the magnetic field volume, within each endcap region of the detector. Muons are measured using gas-ionization detectors embedded in the steel flux-return yoke outside of the solenoid. A detailed description of the CMS detector, its coordinate system, and the main kinematic variables used in the analysis can be found in ref. [1].

Events must satisfy at least one of a set of ee, $\mu \mu$, and e $\mu$ triggers. The ee and $\mu \mu$ triggers collect signal candidate data while the e $\mu$ trigger collects data used in the backgrounddetermination procedure, as described below. These triggers require at least one electron or muon with transverse momentum $p_{\mathrm{T}}>17 \mathrm{GeV}$, and another with $p_{\mathrm{T}}>8 \mathrm{GeV}$. Their efficiencies after event selection $(>90 \%)$ are measured in data and are accounted for in the analysis. The efficiencies of the ee, $\mu \mu$, and e $\mu$ triggers differ by only a few percent in the kinematic range of this search. 


\section{Signal scenarios}

Two classes of signal events are considered, as explained below. Both classes are implemented in the framework of simplified models [8], in which only the targeted production and decay schemes are examined, with all non-participating BSM particles assumed to be too heavy to be relevant.

The first class of signal events targets the production of an edge in the invariant mass spectrum of opposite-sign SF lepton pairs, as expected from the correlated production of these leptons in cascade decays. This class of scenarios is based on the production of a bottom squark-antisquark pair. Each bottom squark $\widetilde{b}$ decays to a bottom quark b and the $\widetilde{\chi}_{2}^{0}$ neutralino. Two specific possibilities are considered. In the first scenario (figure 1 left), the $\widetilde{\chi}_{2}^{0}$ decays to an off-shell $\mathrm{Z}$ boson $\mathrm{Z}^{*}$ and the $\widetilde{\chi}_{1}^{0}$ neutralino, where the $\widetilde{\chi}_{1}^{0}$ is a stable, weakly interacting, lightest SUSY particle (LSP). The $\mathrm{Z}^{*}$ boson decays according to its SM branching fractions, sometimes producing a charged lepton pair $\ell^{+} \ell^{-}(\ell=\mathrm{e}, \mu)$. The mass difference between the $\widetilde{\chi}_{2}^{0}$ and $\widetilde{\chi}_{1}^{0}$, which determines the location of the edge, is fixed to $70 \mathrm{GeV}$. This scenario is referred to as the "fixed-edge" scenario. In the second scenario (figure 1 right), the $\widetilde{\chi}_{2}^{0}$ decays to an on- or off-shell $\mathrm{Z}$ boson and the $\widetilde{\chi}_{1}^{0}$ LSP or according to $\widetilde{\chi}_{2}^{0} \rightarrow \ell \widetilde{\ell}$, with a $50 \%$ probability for each decay. The slepton $\widetilde{\ell}$, i.e., the SUSY partner of the lepton, then decays according to $\widetilde{\ell} \rightarrow \ell \widetilde{\chi}_{1}^{0}$. The considered sleptons are mass-degenerate selectrons and smuons. The mass of the slepton is chosen to lie halfway between the masses of the two neutralinos: $m_{\tilde{\ell}}=m_{\widetilde{\chi}_{1}^{0}}+0.5\left(m_{\widetilde{\chi}_{2}^{0}}-m_{\widetilde{\chi}_{1}^{0}}\right)$. The mass of the $\widetilde{\chi}_{1}^{0}$ is set to $100 \mathrm{GeV}$, with the position of the edge a free parameter in a scan of the mass spectrum. This scenario is referred to as the "slepton-edge" scenario.

The second class of signal events targets the production of an opposite-sign SF lepton pair from the decay of an on-shell $\mathrm{Z}$ boson. This class of scenarios, illustrated in figure 2 , is based on gluino pair production in the context of gauge mediated supersymmetry breaking (GMSB) models [9-11]. Each gluino decays to a quark-antiquark pair and the $\widetilde{\chi}_{1}^{0}$ neutralino. The $\widetilde{\chi}_{1}^{0}$ decays to an on-shell $\mathrm{Z}$ boson and a stable, massless, weakly interacting gravitino LSP. We refer to this scenario as the "GMSB" scenario.

The production of squark and gluino pairs is simulated with the MADGRAPH 5.1.3.30 [12, 13] Monte Carlo (MC) leading-order event generator, including up to two additional partons at the matrix element level. The decays of the squarks, gluinos, and other particles are simulated with the PYTHIA 6.4.22 [14] event generator. The MADGRAPH events are subsequently processed with the PYTHIA program to generate parton showers and account for hadronization. The decay of the $\mathrm{Z}$ boson is handled in PYTHIA. In all the aforementioned scenarios (fixed-edge, slepton-edge, GMSB), the $\mathrm{Z}$ boson decays according to its SM branching fractions. To reduce computational requirements, the detector response is simulated using the CMS fast simulation [15]. Differences in the lepton reconstruction and identification efficiencies between the fast and a "full" simulation, where the full simulation is based on processing through the GEANT4 [16] programs, are corrected using scale factors. The expected signal event rates are normalized to cross sections calculated at the next-to-leading order (NLO) in the strong coupling constant, including the resummation of soft gluon emission at next-to-leading-logarithmic (NLO+NLL) accuracy [17-22]. 

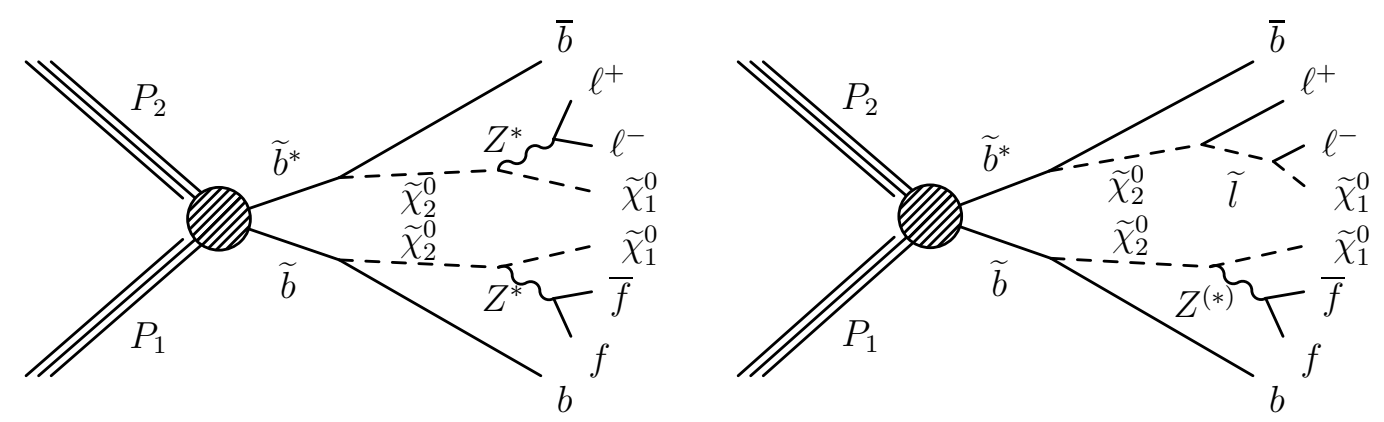

Figure 1. Event diagrams for the (left) "fixed-edge", and (right) "slepton-edge" scenarios, with $\widetilde{\mathrm{b}}$ a bottom squark, $\widetilde{\chi}_{2}^{0}$ the second lightest neutralino, $\widetilde{\chi}_{1}^{0}$ a massive neutralino LSP, and $\widetilde{\ell}$ an electron- or muon-type slepton. For the slepton-edge scenario, the Z boson can be either on- or off-shell, while for the fixed-edge scenario it is off-shell.

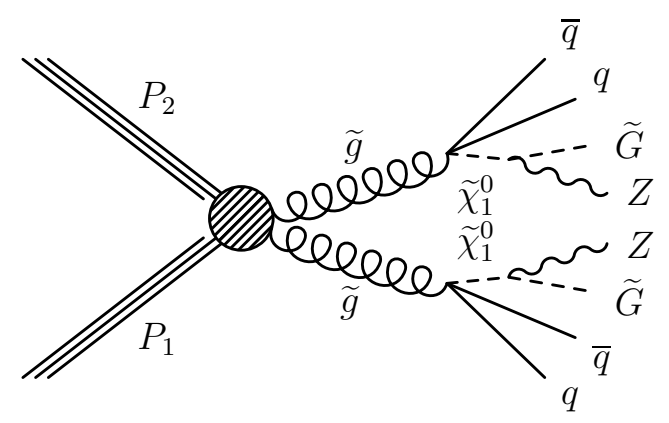

Figure 2. Event diagram for the "GMSB" scenario, with $\widetilde{g}$ a gluino, $\widetilde{\chi}_{1}^{0}$ the lightest neutralino, and $\widetilde{\mathrm{G}}$ a massless gravitino LSP.

\section{Event selection, reconstruction, and search strategy}

We select events with an oppositely charged lepton pair $\left(\mathrm{e}^{+} \mathrm{e}^{-}, \mathrm{e}^{ \pm} \mu^{\mp}\right.$, or $\left.\mu^{+} \mu^{-}\right)$. The leptons are required to have $p_{\mathrm{T}}>20 \mathrm{GeV}$ and $|\eta|<2.4$, where $\eta$ is the pseudorapidity [1]. In events with more than two selected leptons, we choose the two oppositely charged leptons with highest $p_{\mathrm{T}}$. The background estimation techniques employed in this analysis rely, in part, on a symmetry between lepton pairs with the same flavor compared to those with opposite flavor (OF), where OF refers to $\mathrm{e}^{ \pm} \mu^{\mp}$ combinations. It is therefore desirable that the reconstruction efficiencies of electrons [23] and muons [24] be as similar as possible. For this reason, we exclude leptons in the intervals $1.4<|\eta|<1.6$ between the barrel and endcap regions of the detector [1], where the electron and muon reconstruction efficiencies differ significantly. For the SF signal candidate sample, only events with an $\mathrm{e}^{+} \mathrm{e}^{-}$or $\mu^{+} \mu^{-}$ pair are used.

Leptons produced in the decays of low-mass particles, such as hadrons containing $b$ and c quarks, almost always lie in or near jets. The background from these low-mass processes can be suppressed by requiring the leptons to be isolated in space from other particles. A cone of radius $\Delta R \equiv \sqrt{(\Delta \eta)^{2}+(\Delta \phi)^{2}}=0.3$ is constructed around the lepton momentum direction, where $\phi$ is the azimuthal angle. The lepton relative isolation is then quantified 
by the scalar $p_{\mathrm{T}}$ sum of all particle candidates within this cone, excluding the lepton, divided by the lepton $p_{\mathrm{T}}$. The resulting quantity is required to be less than 0.15 . The sum includes a correction to the total energy to account for contributions from extraneous pp interactions within the same or a nearby bunch crossing (pileup). For electrons, the pileup correction is based on the effective area method [25], while for muons it is based on the number of charged hadrons not associated with the primary vertex. The performance of the electron and muon isolation criteria is discussed in refs. [23, 24].

The primary vertex is taken to be the reconstructed vertex with the largest $p_{\mathrm{T}}^{2}$ sum of associated tracks. Leptons with impact parameters larger than $0.2 \mathrm{~mm}$ in the transverse plane or $1 \mathrm{~mm}$ along the beam direction are rejected. As an additional requirement, the two selected leptons must be separated by $\Delta R>0.3$ to avoid systematic effects that arise for isolation requirements in very collinear topologies.

A particle-flow (PF) technique [26] is used to reconstruct jets and missing transverse momentum. All objects reconstructed with the PF algorithm serve as input for jet reconstruction, based on the anti- $k_{\mathrm{T}}$ clustering algorithm [27] with a distance parameter of 0.5 , as implemented in the FASTJET package $[25,28]$. We apply $p_{\mathrm{T}^{-}}$and $\eta$-dependent corrections to account for residual effects of nonuniform detector response. The contribution to the jet energy from pileup is estimated on an event-by-event basis using the jet area method described in ref. [29], and is subtracted from the overall jet $p_{\mathrm{T}}$. Jets are required to have $p_{\mathrm{T}}>40 \mathrm{GeV},|\eta|<3.0$, and to be separated by $\Delta R>0.4$ from all selected leptons. The missing transverse momentum $\vec{p}_{\mathrm{T}}^{\text {miss }}$ is defined as the projection on the plane perpendicular to the beam axis of the negative vector sum of the momenta of all reconstructed PF objects in an event. The magnitude of $\vec{p}_{\mathrm{T}}^{\text {miss }}$ is referred to as $E_{\mathrm{T}}^{\text {miss }}$. The $E_{\mathrm{T}}^{\text {miss }}$ distributions of events in the SF and OF samples for dilepton invariant mass $m_{\ell \ell}>20 \mathrm{GeV}$ and number of jets $N_{\text {jets }} \geq 2$ are shown in figure 3 .

The event selection criteria are motivated by the expectation that BSM signal events, involving the production of new heavy particles, generally have larger jet multiplicity and $E_{\mathrm{T}}^{\text {miss }}$ than background events, which primarily arise from top quark-antiquark $(\mathrm{t} \overline{\mathrm{t}})$ and Drell-Yan (DY) processes. The large value of $E_{\mathrm{T}}^{\text {miss }}$ expected in signal events is due to the weakly interacting LSP particles, which escape without detection.

In the search for an edge, we define two signal regions: either $N_{\text {jets }} \geq 2$ and $E_{\mathrm{T}}^{\text {miss }}>$ $150 \mathrm{GeV}$, or $N_{\text {jets }} \geq 3$ and $E_{\mathrm{T}}^{\text {miss }}>100 \mathrm{GeV}$. For both regions, we separately consider events in which both leptons satisfy $\left|\eta_{\text {lep }}\right|<1.4$ ("central" signal region) and events in which at least one lepton satisfies $1.6<\left|\eta_{\text {lep }}\right|<2.4$ ("forward" signal region). The motivation for the distinction between the central and forward regions is that for BSM production through the decay of heavy resonances, the final-state leptons and jets are expected to be more centrally distributed than for the SM backgrounds. Two methods are used to search for an edge signature. In the first method, a search for an edge is performed in the range $20<m_{\ell \ell}<300 \mathrm{GeV}$ by fitting the signal and background hypotheses to data, as described in section 6 . In the second method, based on a direct comparison of event counts, with no assumption about the shapes of the signal and background distributions, we select three regions, $20<m_{\ell \ell}<70 \mathrm{GeV}, 81<m_{\ell \ell}<101 \mathrm{GeV}$, and $m_{\ell \ell}>120 \mathrm{GeV}$, denoted the "lowmass", "on-Z", and "high-mass" regions, respectively. For this "counting experiment", the integrated yield in each region is compared to the corresponding background prediction. 

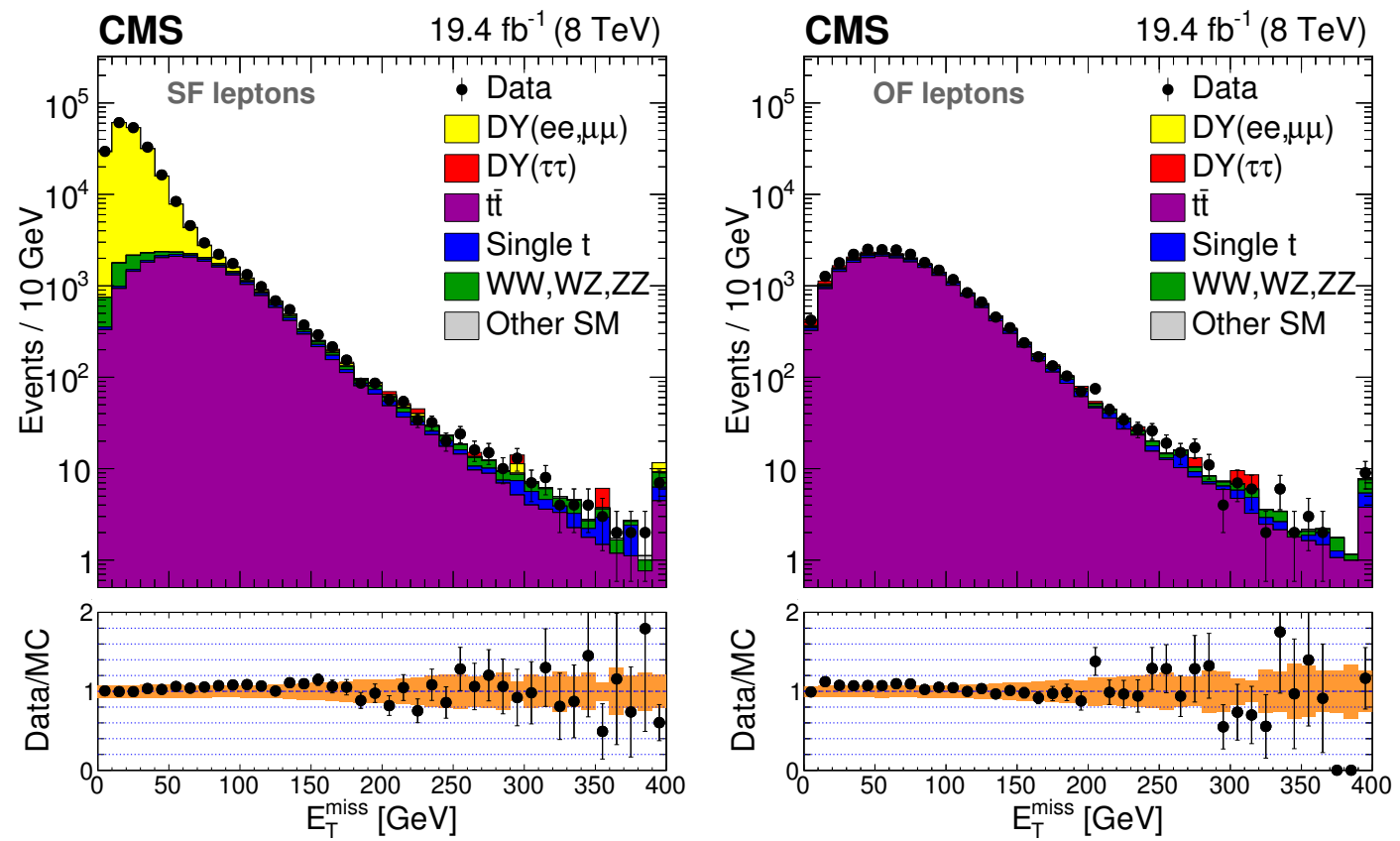

Figure 3. The $E_{\mathrm{T}}^{\text {miss }}$ distributions of events in the SF (left) and OF (right) samples for $m_{\ell \ell}>$ $20 \mathrm{GeV}$ and $N_{\text {jets }} \geq 2$ in comparison with predictions for the SM background from the MC generators described in section 4 . In the ratio panel below each plot, the error bars on the black points show the statistical uncertainties of the data and MC samples, while the shaded band indicates the MC statistical and systematic uncertainties added in quadrature. The rightmost bins contain the overflow.

In the search for BSM events with an on-shell $\mathrm{Z}$ boson, we perform a dedicated counting experiment in the region $81<m_{\ell \ell}<101 \mathrm{GeV}$, based on the distribution of $E_{\mathrm{T}}^{\text {miss }}$. For this study, two inclusive bins in the number of jets are defined: $N_{\text {jets }} \geq 2$ and $N_{\text {jets }} \geq 3$. Events are examined in exclusive bins of $E_{\mathrm{T}}^{\text {miss }}$ as described in section 7 .

While the main SM backgrounds are estimated using data control samples, simulated $\mathrm{MC}$ events are used to evaluate smaller sources of background. The simulation is also used to estimate uncertainties. Simulated samples of DY + jets, t $\bar{t}, \mathrm{~W}+$ jets, VV, and $\mathrm{t} \overline{\mathrm{t}} \mathrm{V}(\mathrm{V}=\mathrm{W}, \mathrm{Z})$ events are generated with the MADGRAPH 5.1.3.30 event generator. The $\mathrm{DY}+$ jets sample considers events with dilepton invariant masses as low as $10 \mathrm{GeV}$, as well as decays to the $\tau \tau$ channel. The matrix element calculation performed with MADGRAPH 5.1.3.30 is then interfaced to the PYTHIA 6.4.22 program for the description of parton showering and hadronization, analogous to the procedure used for the signal samples. The detector response in these samples is simulated with a GEANT4 model [16] of the CMS detector. The simulated events are reconstructed and analyzed with the same software used to process data. In the simulation, multiple pp interactions are superimposed on the hard collision, and the simulated samples are reweighted to reflect the beam conditions, taking into account the total inelastic pp cross section at the LHC. Corrections are applied to account for the differences between simulation and data in the trigger and reconstruction efficiencies. The simulated sample yields are normalized to an integrated luminosity 
of $19.4 \mathrm{fb}^{-1}$ using NLO inclusive cross sections, except for the DY + jets and $\mathrm{W}+$ jets samples, where next-to-next-to-leading order calculations [30] are used.

\section{$5 \quad$ Background estimates}

The principal SM backgrounds are divided into two categories. Backgrounds that produce OF pairs $\left(\mathrm{e}^{+} \mu^{-}, \mathrm{e}^{-} \mu^{+}\right)$as often as SF pairs $\left(\mathrm{e}^{+} \mathrm{e}^{-}, \mu^{+} \mu^{-}\right)$are referred to as flavorsymmetric (FS) backgrounds. This category is dominated by t $\overline{\mathrm{t}}$ processes. Drell-Yan events form the second principal background category. The FS background estimate accounts also for $\mathrm{WW}, \mathrm{Z} / \gamma^{*}(\rightarrow \tau \tau)$, and tW single-top quark production, as well as for backgrounds due to leptons from hadron decays and from hadrons misidentified as leptons. Contributions from $t \bar{t}+\mathrm{X}$, with $\mathrm{X}$ a $\mathrm{W}, \mathrm{Z}$, or Higgs boson, have been studied in the simulation and found to be negligible.

The missing transverse momentum in DY + jets events arises primarily from jet energy resolution and reconstruction effects. Contributions from SM WZ and ZZ processes, which might include genuine $E_{\mathrm{T}}^{\mathrm{miss}}$, are incorporated into the $\mathrm{DY}+$ jets background estimates.

\subsection{Flavor-symmetric backgrounds}

The contribution of FS background events to the signal regions is determined using a control sample defined by OF events that satisfy the full event selection. The OF yields are multiplied by a factor $R_{\mathrm{SF} / \mathrm{OF}}$ to account for efficiency differences in the selection of dilepton pairs in the SF and OF samples. Two methods are used to evaluate the $R_{\mathrm{SF}} \mathrm{OF}$ factor, as explained below. We also determine corresponding factors $R_{\mathrm{ee} / \mathrm{OF}}$ and $R_{\mu \mu / \mathrm{OF}}$ for the individual SF terms.

The first method, referred to as the "factorization" method, uses a data control sample to determine the SF-to-OF ratio of reconstruction efficiencies, and a second data control sample to determine the corresponding ratio of trigger efficiencies. The reconstruction efficiency ratio is evaluated using a large DY + jets data control sample selected by requiring $60<m_{\ell \ell}<120 \mathrm{GeV}, N_{\text {jets }} \geq 2$, and $E_{\mathrm{T}}^{\text {miss }}<50 \mathrm{GeV}$. The measured quantity is $r_{\mu \mathrm{e}}=\sqrt{N_{\mu \mu} / N_{\mathrm{ee}}}$, which represents a ratio of the muon-to-electron reconstruction efficiency uncorrected for trigger efficiencies. The extrapolation of $r_{\mu \mathrm{e}}$ into the central (forward) signal region is studied using data. A 10\% (20\%) systematic uncertainty is assigned to account for the observed dependencies. The ratio $R_{\mathrm{T}}$ of the trigger efficiencies in SF and OF events is determined using a data control sample selected with a trigger based on $H_{\mathrm{T}}$, which is the scalar sum of jet $p_{\mathrm{T}}$ values for jets with $p_{\mathrm{T}}>40 \mathrm{GeV}$. The sample is selected by requiring $H_{\mathrm{T}}>200 \mathrm{GeV}, m_{\ell \ell}>20 \mathrm{GeV}$, and excluding events with $N_{\text {jets }}=2$ and $E_{\mathrm{T}}^{\text {miss }}>100 \mathrm{GeV}$. The latter two requirements ensure that this control sample is uncorrelated with the control sample used to determine the reconstruction efficiencies. A 5\% uncertainty is assigned to the efficiency of each dilepton trigger $\left(\mathrm{e}^{+} \mathrm{e}^{-}, \mu^{+} \mu^{-}, \mathrm{e}^{ \pm} \mu^{\mp}\right)$ to account for the observed dependence of these efficiencies on $m_{\ell \ell}, E_{\mathrm{T}}^{\text {miss }}$, and $N_{\text {jets. }}$. The full correction to the OF event rate is $R_{\mathrm{SF} / \mathrm{OF}}=0.5\left(r_{\mu \mathrm{e}}+r_{\mu \mathrm{e}}^{-1}\right) R_{\mathrm{T}}$, with $R_{\mathrm{T}}=\sqrt{\epsilon_{\mathrm{ee}}^{\text {trig }} \epsilon_{\mu \mu}^{\text {trig }}} / \epsilon_{\mathrm{e} \mu}^{\text {trig }}$.

The second method, referred to as the "control-region" method, directly measures the $R_{\mathrm{SF} / \mathrm{OF}}$ factors from the ratio of the $\mathrm{e}^{+} \mathrm{e}^{-}, \mu^{+} \mu^{-}$, or combined SF yields with the OF 


\begin{tabular}{|l|c|c|}
\hline & Central & Forward \\
\hline \multicolumn{3}{|c|}{ Factorization method } \\
\hline$R_{\mathrm{SF} / \mathrm{OF}}$ & $1.03 \pm 0.01 \pm 0.06$ & $1.11 \pm 0.04 \pm 0.08$ \\
$R_{\mathrm{ee} / \mathrm{OF}}$ & $0.47 \pm 0.01 \pm 0.06$ & $0.46 \pm 0.02 \pm 0.10$ \\
$R_{\mu \mu / \mathrm{OF}}$ & $0.56 \pm 0.01 \pm 0.07$ & $0.65 \pm 0.03 \pm 0.14$ \\
\hline$r_{\mu \mathrm{e}}$ & $1.09 \pm 0.00 \pm 0.11$ & $1.18 \pm 0.00 \pm 0.24$ \\
$R_{\mathrm{T}}$ & $1.03 \pm 0.01 \pm 0.06$ & $1.10 \pm 0.04 \pm 0.07$ \\
\hline \multicolumn{3}{|c|}{ Control-region method } \\
\hline$R_{\mathrm{SF} / \mathrm{OF}}$ & $0.99 \pm 0.05 \pm 0.02$ & $1.11 \pm 0.11 \pm 0.03$ \\
$R_{\mathrm{ee} / \mathrm{OF}}$ & $0.44 \pm 0.03 \pm 0.01$ & $0.49 \pm 0.06 \pm 0.02$ \\
$R_{\mu \mu / \mathrm{OF}}$ & $0.55 \pm 0.03 \pm 0.01$ & $0.62 \pm 0.07 \pm 0.02$ \\
\hline$r_{\mu \mathrm{e}}$ & $1.12 \pm 0.04$ (stat) & $1.12 \pm 0.08$ (stat) \\
$R_{\mathrm{T}}$ & $0.98 \pm 0.05$ (stat) & $1.11 \pm 0.11$ (stat) \\
\hline \multicolumn{3}{|c|}{ Combined } \\
\hline$R_{\mathrm{SF} / \mathrm{OF}}$ & $1.00 \pm 0.04$ & $1.11 \pm 0.07$ \\
$R_{\mathrm{ee} / \mathrm{OF}}$ & $0.45 \pm 0.03$ & $0.48 \pm 0.05$ \\
$R_{\mu \mu / \mathrm{OF}}$ & $0.55 \pm 0.03$ & $0.63 \pm 0.07$ \\
\hline
\end{tabular}

Table 1. Results for $R_{\mathrm{SF} / \mathrm{OF}}$ in the signal regions. The results of the two methods are shown with statistical and systematic uncertainties, while the uncertainties for the combined values are a combination of the statistical and systematic terms. The values of $r_{\mu \mathrm{e}}$ and $R_{\mathrm{T}}$ listed for the controlregion method are not used directly in the analysis and are listed for purposes of comparison only.

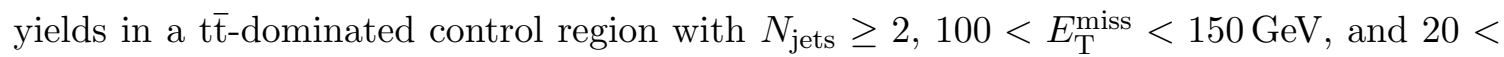
$m_{\ell \ell}<70 \mathrm{GeV}$. Differences between the factors obtained in this control region and in the signal regions are studied with the t $\overline{\mathrm{t}}$ simulation. No difference is observed within the MC statistical uncertainty, which is used to define the corresponding systematic uncertainty. The size of this uncertainty is found to be $2 \%(3-4 \%)$ in the central (forward) region.

As the two methods rely on uncorrelated control samples, and the results agree within their uncertainties, the final results for $R_{\mathrm{SF} / \mathrm{OF}}$ are obtained by taking the weighted average of the results from the two methods, propagating the uncertainties. The results are shown in table 1. The FS background is then given by the number of events in the OF control samples multiplied by the corresponding $R_{\mathrm{SF} / \mathrm{OF}}$ factor. It is seen that the values of the $R_{\mathrm{SF} / \mathrm{OF}}$ ratios are consistent with unity within the uncertainties. We find that the variation of $R_{\mathrm{SF} / \mathrm{OF}}$ for increasing $m_{\ell \ell}, N_{\text {jets }}$, and $E_{\mathrm{T}}^{\text {miss }}$ lies within the assigned uncertainty. We thus use the same value of $R_{\mathrm{SF} / \mathrm{OF}}$ for all signal regions. 


\subsection{SM Drell-Yan background}

In the search for an edge based on a fit, the DY+jets background is determined as described in section 6. For the counting experiment method, the DY background is determined using the jet-Z balance (JZB) and $E_{\mathrm{T}}^{\text {miss }}$-template methods [5], as described in this section.

The JZB is a measure of the imbalance between the $p_{\mathrm{T}}$ of the $\mathrm{Z} / \gamma^{*}$ boson and the $p_{\mathrm{T}}$ of the recoiling hadronic system in DY + jets. The JZB is defined as the scalar difference between the $p_{\mathrm{T}}$ of the $\mathrm{Z} / \gamma^{*}$ and the net $p_{\mathrm{T}}$ of the recoiling hadronic system. Standard model DY + jets events equally populate negative and positive values of JZB, because non-zero JZB in these events arises from jet energy resolution effects, whereas in BSM and $t \bar{t}$ events, which contain genuine $E_{\mathrm{T}}^{\text {miss }}$, JZB can be very asymmetric towards positive values because of the correlated production of the lepton pair and the undetected particles. Events with negative values of JZB mainly arise from DY + jets processes, with a small contribution from $t \bar{t}$ production. The number of $t \overline{\mathrm{t}}$ events that contribute negative JZB values is determined using the corresponding results for OF events. The t $\bar{t}$ contribution is then subtracted from the number of negative JZB events in the SF sample to estimate the DY+jets background. Uncertainties arising from imperfect knowledge of the $R_{\mathrm{SF} / \mathrm{OF}}$ factor (table 1 ) when subtracting the $t \bar{t}$ contribution are propagated to the final DY + jets estimate. This method also accounts for processes with DY $\rightarrow \ell^{ \pm} \ell^{\mp}+X$, where $X$ denotes other particles that might be present in the final state. The systematic uncertainty in the assumption that DY + jets events equally populate positive and negative values of JZB is evaluated in simulation by comparing the $E_{\mathrm{T}}^{\text {miss }}$ distributions of events with JZB $<0$ and $>0$. A systematic uncertainty of $20 \%$ is assigned to account for possible differences, dominated by the limited statistical precision of the MC sample.

The $E_{\mathrm{T}}^{\text {miss }}$-template method relies on a data control sample consisting of events with photons and jets to evaluate the DY + jets background in a high $E_{\mathrm{T}}^{\mathrm{miss}}$ signal region. For both $\mathrm{DY}+$ jets and $\gamma+$ jets events, large values of $E_{\mathrm{T}}^{\text {miss }}$ arise from the mismeasurement of jet $p_{\mathrm{T}}$ values. The $E_{\mathrm{T}}^{\mathrm{miss}}$ distribution for $\mathrm{DY}+$ jets events can thus be evaluated using $\gamma+$ jets events selected with similar kinematic requirements. After selection, the $N_{\text {jets }}$, $H_{\mathrm{T}}$, and boson $p_{\mathrm{T}}$ distributions of the $\gamma+$ jets sample are reweighted to match those of the DY + jets sample. The systematic uncertainty is determined in simulation by applying this reweighting to a $\gamma+$ jets $\mathrm{MC}$ sample and comparing the reweighted $E_{\mathrm{T}}^{\text {miss }}$ spectrum to that in a DY + jets MC sample. The uncertainty is taken as the larger of the difference between the two samples or the MC statistical uncertainty. The assigned uncertainty is $4 \%$ for $E_{\mathrm{T}}^{\text {miss }}<60 \mathrm{GeV}, 15 \%$ for $60<E_{\mathrm{T}}^{\text {miss }}<200 \mathrm{GeV}, 34 \%$ for $200<E_{\mathrm{T}}^{\text {miss }}<300 \mathrm{GeV}$, and $100 \%$ for $E_{\mathrm{T}}^{\text {miss }}>300 \mathrm{GeV}$. The uncertainty in the last two $E_{\mathrm{T}}^{\text {miss }}$ bins is mostly due to the MC statistical uncertainty. Uncertainties are also assigned to account for the difference in the number of pileup interactions in the triggered DY + jets and $\gamma+$ jets events $(1-3 \%$, increasing with $\left.E_{\mathrm{T}}^{\text {miss }}\right)$ and the purity of the photon selection (1-5\%, increasing with $E_{\mathrm{T}}^{\text {miss }}$ ).

With the $E_{\mathrm{T}}^{\text {miss }}$-template method, the backgrounds from WZ, ZZ, and other rare SM processes are estimated using simulation. An uncertainty of $50 \%$ is assigned to the $\mathrm{WZ}$ and ZZ backgrounds based on comparisons with data in orthogonal control samples selected by requiring $N_{\text {jets }} \geq 2$ and either exactly three leptons (WZ control sample) or exactly four 
leptons (ZZ control sample). For the other rare backgrounds, which include events with $\mathrm{t} \overline{\mathrm{tZ}}$, ZZZ, ZZW, and ZWW production, an uncertainty of $50 \%$ is similarly assigned.

The two methods are used to estimate the yield of SM DY events in the on-Z region, with all other selection criteria the same as for signal events. Since the two methods use uncorrelated samples to describe the high- $E_{\mathrm{T}}^{\text {miss }}$ tail of SM DY + jets production, the DY background estimate in the on- $Z$ region is taken to be the weighted average of the two estimates. The individual results are consistent with the weighted average within their uncertainties.

This estimate of the DY + jets background is extrapolated outside on- $\mathrm{Z}$ region using the ratio $R_{\text {out/in }}$. It is measured in data as the event yield outside the on-Z region divided by the yield in the on-Z region, for the dilepton invariant mass distribution in SF events with $E_{\mathrm{T}}^{\text {miss }}<50 \mathrm{GeV}$ and $N_{\text {jets }} \geq 2$, after subtraction of the FS backgrounds. This distribution is almost entirely composed of lepton pairs from DY processes, making it suitable for the determination of $R_{\text {out } / \text { in }}$. A systematic uncertainty of $25 \%$ is assigned to $R_{\text {out/in }}$ to account for possible biases introduced by the different selection criteria used for the signal and control regions, and for differences in the value of $R_{\text {out/in }}$ between electrons and muons.

\section{Kinematic fit}

The search for an edge based on the fit method is performed using a simultaneous extended unbinned maximum likelihood fit to the dilepton mass distributions of $\mathrm{e}^{+} \mathrm{e}^{-}, \mu^{+} \mu^{-}$, and $\mathrm{e}^{ \pm} \mu^{\mp}$ events. The likelihood model contains three components: (a) an FS background component, (b) a DY background component, and (c) a signal component.

The FS background is described using a model with three regions: a low-mass region modeling the rising distribution shaped by lepton acceptance requirements, a transition region, and a high-mass region in which the distribution falls exponentially. The probability density functions describing the three regions are:

$$
\mathcal{P}_{F S}\left(m_{\ell \ell}\right)=\left\{\begin{array}{lll}
\mathcal{P}_{F S, 1}\left(m_{\ell \ell}\right)=c_{1} m_{\ell \ell}^{\alpha} & \text { if } & 20 \mathrm{GeV}<m_{\ell \ell}<m_{\ell \ell}^{(1)}, \\
\mathcal{P}_{F S, 2}\left(m_{\ell \ell}\right)=\sum_{i=1}^{4} c_{2, i} m_{\ell \ell}^{i-1} & \text { if } & m_{\ell \ell}^{(1)}<m_{\ell \ell}<m_{\ell \ell}^{(2)} \\
\mathcal{P}_{F S, 3}\left(m_{\ell \ell}\right)=c_{3} \mathrm{e}^{-\beta m_{\ell \ell}} & \text { if } & m_{\ell \ell}^{(2)}<m_{\ell \ell}<300 \mathrm{GeV}
\end{array}\right.
$$

where $m_{\ell \ell}^{(1)}$ and $m_{\ell \ell}^{(2)}$ define the boundaries between the regions. Because of the requirement that the function and its derivative both be continuous, the FS model is left with five independent parameters plus the normalization.

The DY background is modeled with the sum of an exponential function, which describes the low-mass rise, and a Breit-Wigner function with a mean and width set to the nominal $\mathrm{Z}$ boson values [31], which accounts for the on- $\mathrm{Z}$ lineshape. To account for the experimental resolution, the Breit-Wigner function is convolved with a double-sided 
Crystal-Ball [32] function $\mathcal{P}_{\mathrm{DSCB}}\left(m_{\ell \ell}\right)$ :

$$
\mathcal{P}_{\mathrm{DSCB}}\left(m_{\ell \ell}\right)=\left\{\begin{array}{lll}
A_{1}\left(B_{1}-\frac{m_{l l}-\mu_{\mathrm{CB}}}{\sigma_{\mathrm{CB}}}\right)^{-n_{1}} & \text { if } & \frac{m_{l l}-\mu_{\mathrm{CB}}}{\sigma_{\mathrm{CB}}}<-\alpha_{1}, \\
\exp \left(-\frac{\left(m_{l l}-\mu_{\mathrm{CB}}\right)^{2}}{2 \sigma_{\mathrm{CB}}^{2}}\right) & \text { if } & -\alpha_{1}<\frac{m_{l l}-\mu_{\mathrm{CB}}}{\sigma_{\mathrm{CB}}}<\alpha_{2}, \\
A_{2}\left(B_{2}+\frac{m_{l l}-\mu_{\mathrm{CB}}}{\sigma_{\mathrm{CB}}}\right)^{-n_{2}} & \text { if } & \frac{m_{l l}-\mu_{\mathrm{CB}}}{\sigma_{\mathrm{CB}}}>\alpha_{2},
\end{array}\right.
$$

where

$$
A_{i}=\left(\frac{n_{i}}{\left|\alpha_{i}\right|}\right)^{n_{i}} \exp \left(-\frac{\left|\alpha_{i}\right|^{2}}{2}\right) \quad \text { and } \quad B_{i}=\frac{n_{i}}{\left|\alpha_{i}\right|}-\left|\alpha_{i}\right| .
$$

The full model for the on-Z DY lineshape is thus:

$$
\mathcal{P}_{\mathrm{DY}, \text { on-Z }}\left(m_{\ell \ell}\right)=\int \mathcal{P}_{\mathrm{DSCB}}\left(m_{\ell \ell}\right) \mathcal{P}_{\mathrm{BW}}\left(m_{\ell \ell}-m^{\prime}\right) \mathrm{d} m^{\prime} .
$$

The signal component is described by a triangular shape, convolved with a Gaussian distribution to account for the experimental resolution:

$$
\mathcal{P}_{S}\left(m_{\ell \ell}\right)=\frac{1}{\sqrt{2 \pi \sigma_{\ell \ell}}} \int_{0}^{m_{\ell \ell}^{\text {edge }}} y \exp \left(-\frac{\left(m_{\ell \ell}-y\right)^{2}}{2 \sigma_{\ell \ell}^{2}}\right) \mathrm{d} y .
$$

As a preliminary step, a fit is performed separately for electrons and muons in the DY-enriched control region (the same control region as described for $R_{\text {out/in }}$ in section 5.2) to determine the shape of backgrounds containing a $\mathrm{Z}$ boson. In systematic studies of the fit, the DY shape parameter that models the exponentially falling spectrum of the virtual photon is varied by $\pm 25 \%$. The total effect on the fitted signal yield is found to be negligible. The parameters of the DY shape are then fixed and only the normalizations of these backgrounds are free parameters in the fit. The nominal fit is applied simultaneously in the signal region to the dilepton invariant mass distributions in the $\mathrm{e}^{+} \mathrm{e}^{-}, \mu^{+} \mu^{-}$, and $\mathrm{e}^{ \pm} \mu^{\mp}$ samples. Therefore the model for the FS background is the same for the SF and OF events. The $R_{\mathrm{SF} / \mathrm{OF}}$ factors, in the central and forward regions, are treated as nuisance parameters, parametrized by Gaussian distributions with a mean value and standard deviation given by the values of $R_{\mathrm{SF} / \mathrm{OF}}$ and their uncertainties (table 1 ). The fit is carried out in the central and forward regions simultaneously, with the position of the edge as the only shared parameter. Therefore the signal model has three free parameters: the fitted signal yield in the central and forward regions separately, and the position of the edge.

\section{Results}

The dilepton mass distributions and the results of the fit in the central and forward signal regions are shown in figure 4 . Table 2 presents a summary of the fit results. A signal yield of $126 \pm 41(22 \pm 20)$ events is obtained when evaluating the signal hypothesis in the central (forward) region, with an edge located at $78.7 \pm 1.4 \mathrm{GeV}$. The p-value, evaluated using $-2 \ln Q$, is 0.009 , where $Q$ denotes the ratio of the fitted likelihood value for the 


\begin{tabular}{|l|cc|}
\hline & Central & Forward \\
\hline Drell-Yan & $158 \pm 23$ & $71 \pm 15$ \\
OF yield & $2270 \pm 44$ & $745 \pm 25$ \\
$R_{\text {SF } / O F}$ & $1.03 \pm 0.03$ & $1.02 \pm 0.05$ \\
Signal events & $126 \pm 41$ & $22 \pm 20$ \\
$m_{\ell \ell}^{\text {edge }}$ & $78.7 \pm 1.4 \mathrm{GeV}$ \\
\hline Local significance & \multicolumn{2}{|c|}{$2.4 \sigma$} \\
\hline
\end{tabular}

Table 2. Results of the unbinned maximum likelihood fit for event yields in the signal regions. The quoted uncertainties are calculated using the MINOS [33] program and account for both statistical and systematic sources.

signal-plus-background hypothesis to the background-only hypothesis for the case where the edge position is fixed to the observed value. This p-value is interpreted as the one-sided tail probability of a Gaussian distribution and corresponds to an excess in the observed number of events compared to the SM background estimate of 2.4 standard deviations.

As cross-checks, we tested alternative shapes for the FS background, specifically the sum of three Gaussian distributions, and binned and smoothed histograms taken from the OF events. In all cases, the results were found to be consistent with the nominal results.

Besides the fit described in section 6 , we perform a counting experiment in the mass windows $20<m_{\ell \ell}<70 \mathrm{GeV}, 81<m_{\ell \ell}<101 \mathrm{GeV}$, and $m_{\ell \ell}>120 \mathrm{GeV}$, with no assumption about a particular signal shape, as mentioned in section 4 . Figure 5 shows the invariant mass distributions for the signal candidate sample and the estimated background. For the background prediction, the $\mathrm{OF}$ yield in the signal mass window is multiplied by the $R_{\mathrm{SF} / \mathrm{OF}}$ factor, and the background prediction for backgrounds containing a $\mathrm{Z}$ boson in the on- $\mathrm{Z}$ region by the $R_{\text {out/in }}$ factor, as described in sections 5.1 and 5.2 .

The results are summarized in table 3 . The significance of the excess in the observed number of events with respect to the estimated number of SM background events is evaluated using a profile likelihood asymptotic approximation [34]. The local significance of the excess in the central low-mass region, where the largest deviation is found, is 2.6 standard deviations. Note that the signal regions were defined before the data sample was examined, and that the low-mass region $\left(20<m_{\ell \ell}<70 \mathrm{GeV}\right)$ does not include events between $70 \mathrm{GeV}$ and the best-fit value for the location of the edge $\left(m_{\ell \ell}=78.7 \mathrm{GeV}\right)$. The flavor of the $\ell^{+} \ell^{-}$pair was studied in the counting experiment. Within the statistical uncertainty and accounting for differences in the reconstruction efficiencies, the electron and the muon channels are found to contribute evenly to the excess. Further studies of the excess in the low-mass region do not yield evidence for a neglected systematic term. The excess is observed predominantly in events with at least one identified bottom quark jet (b jet) and diminishes if a veto on the presence of $a b$ jet is applied. To identify $b$ jets, we use the CSV algorithm at the medium working point [35]. 

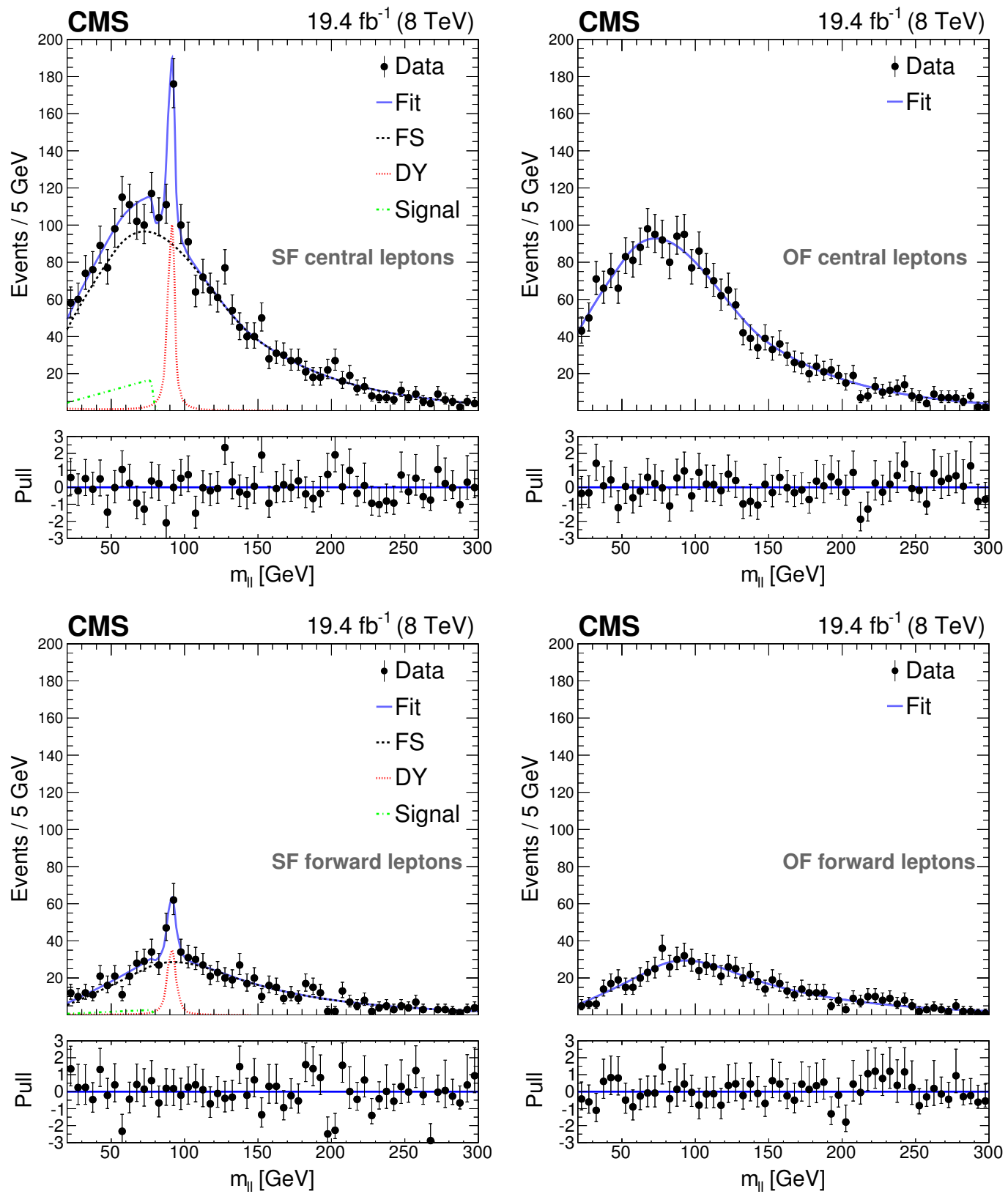

Figure 4. Fit results for the signal-plus-background hypothesis in comparison with the measured dilepton mass distributions, in the central (top) and forward (bottom) regions, projected on the same-flavor (left) and opposite-flavor (right) event samples. The combined fit shape is shown as a blue, solid line. The individual fit components are indicated by dashed lines. The flavor-symmetric (FS) background is displayed with a black dashed line. The Drell-Yan (DY) background is displayed with a red dashed line. The extracted signal component is displayed with a green dashed line. The lower plots show the pull distributions, defined as $\left(N_{\text {data }}-N_{\text {fit }}\right) / \sigma_{\text {data }}$.

Figure 6 presents a comparison of the data and the SM simulation in the central region. Expectations for the fixed-edge bottom-squark pair-production scenario of figure 1 (left), with mass combinations $\left(m_{\widetilde{\mathrm{b}}}, m_{\widetilde{\chi}_{2}^{0}}\right)=(225,150) \mathrm{GeV},(350,275) \mathrm{GeV}$, and $(400,150) \mathrm{GeV}$ 

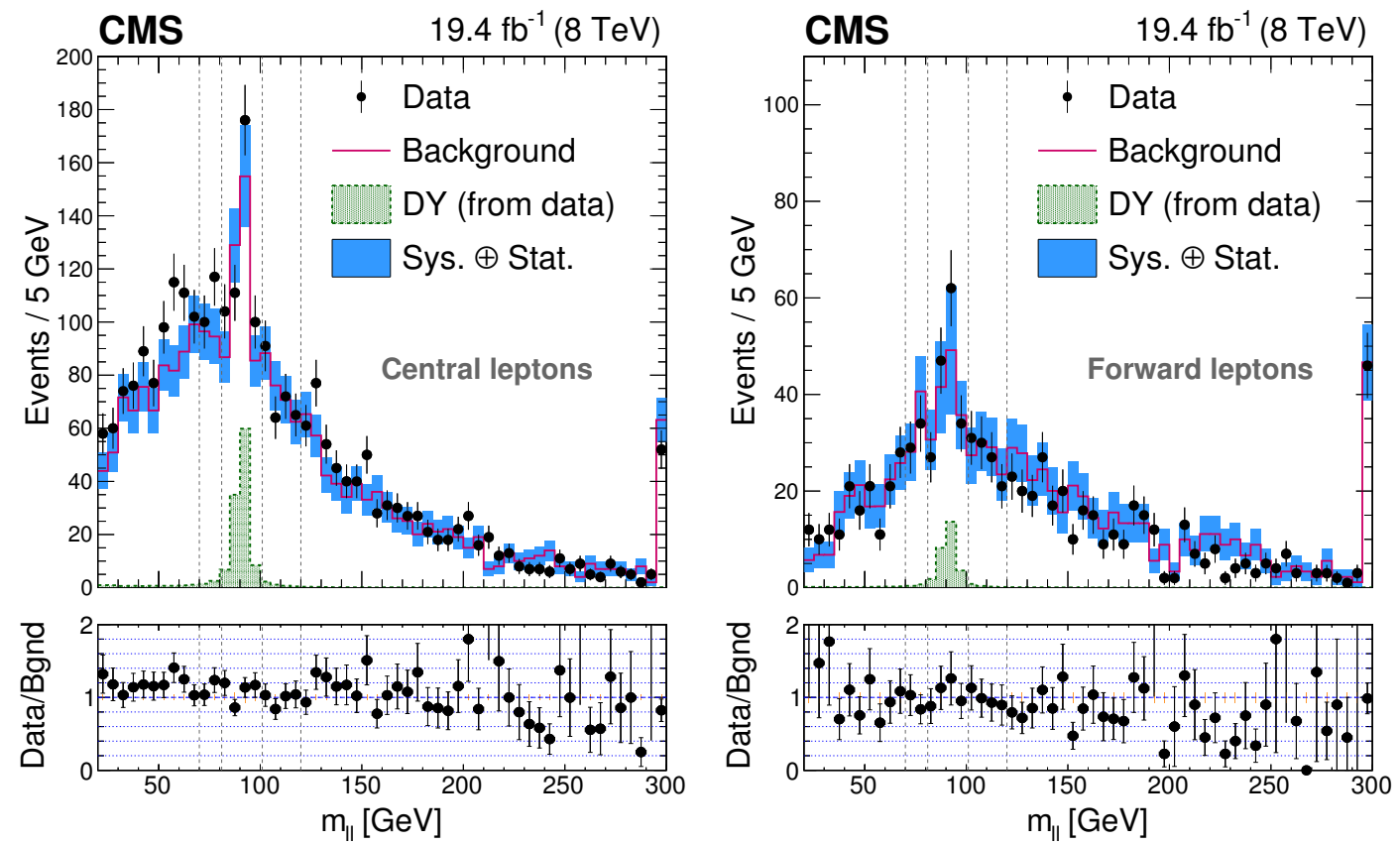

Figure 5. Comparison between the observed and estimated SM background dilepton mass distributions in the (left) central and (right) forward regions, where the SM backgrounds are evaluated from control samples (see text) rather than from a fit. The rightmost bins contain the overflow. The vertical dashed lines denote the boundaries of the low-mass, on-Z, and high-mass regions. The lower plots show the ratio of the data to the predicted background. The error bars for both the main and lower plots include both statistical and systematic uncertainties.

\begin{tabular}{|l|cc|cc|cc|}
\hline & \multicolumn{2}{|c}{ Low-mass } & \multicolumn{2}{c|}{ On-Z } & \multicolumn{2}{c|}{ High-mass } \\
\hline & Central & Forward & Central & Forward & Central & Forward \\
\hline Observed & 860 & 163 & 487 & 170 & 818 & 368 \\
\hline Flavor-symmetric & $722 \pm 27 \pm 29$ & $155 \pm 13 \pm 10$ & $355 \pm 19 \pm 14$ & $131 \pm 12 \pm 8$ & $768 \pm 28 \pm 31$ & $430 \pm 22 \pm 27$ \\
Drell-Yan & $8.2 \pm 2.6$ & $2.5 \pm 1.0$ & $116 \pm 21$ & $42 \pm 9$ & $2.5 \pm 0.8$ & $1.1 \pm 0.4$ \\
\hline Total estimated & $730 \pm 40$ & $158 \pm 16$ & $471 \pm 32$ & $173 \pm 17$ & $771 \pm 42$ & $431 \pm 35$ \\
\hline Observed-estimated & $130_{-49}^{+48}$ & $5_{-20}^{+20}$ & $16_{-38}^{+37}$ & $-3_{-21}^{+20}$ & $47_{-50}^{+49}$ & $-62_{-39}^{+37}$ \\
\hline Significance & $2.6 \sigma$ & $0.3 \sigma$ & $0.4 \sigma$ & $<0.1 \sigma$ & $0.9 \sigma$ & $<0.1 \sigma$ \\
\hline
\end{tabular}

Table 3. Results of the edge-search counting experiment for event yields in the signal regions. The statistical and systematic uncertainties are added in quadrature, except for the flavor-symmetric backgrounds. Low-mass refers to $20<m_{\ell \ell}<70 \mathrm{GeV}$, on-Z to $81<m_{\ell \ell}<101 \mathrm{GeV}$, and high-mass to $m_{\ell \ell}>120 \mathrm{GeV}$.

for the bottom squark and $\widetilde{\chi}_{2}^{0}$, are also shown. The first scenario presents the illustration of a model that can easily be excluded, while the other two present examples of models that are consistent with our data.

The results from the dedicated on-Z counting experiment mentioned in section 4 are presented in tables 4 and 5 for events with $N_{\text {jets }} \geq 2$ and $N_{\text {jets }} \geq 3$, respectively. The corresponding $E_{\mathrm{T}}^{\mathrm{miss}}$ distributions are shown in figure 7 . The data are seen to agree with the SM predictions across the full $E_{\mathrm{T}}^{\mathrm{miss}}$ spectrum. 

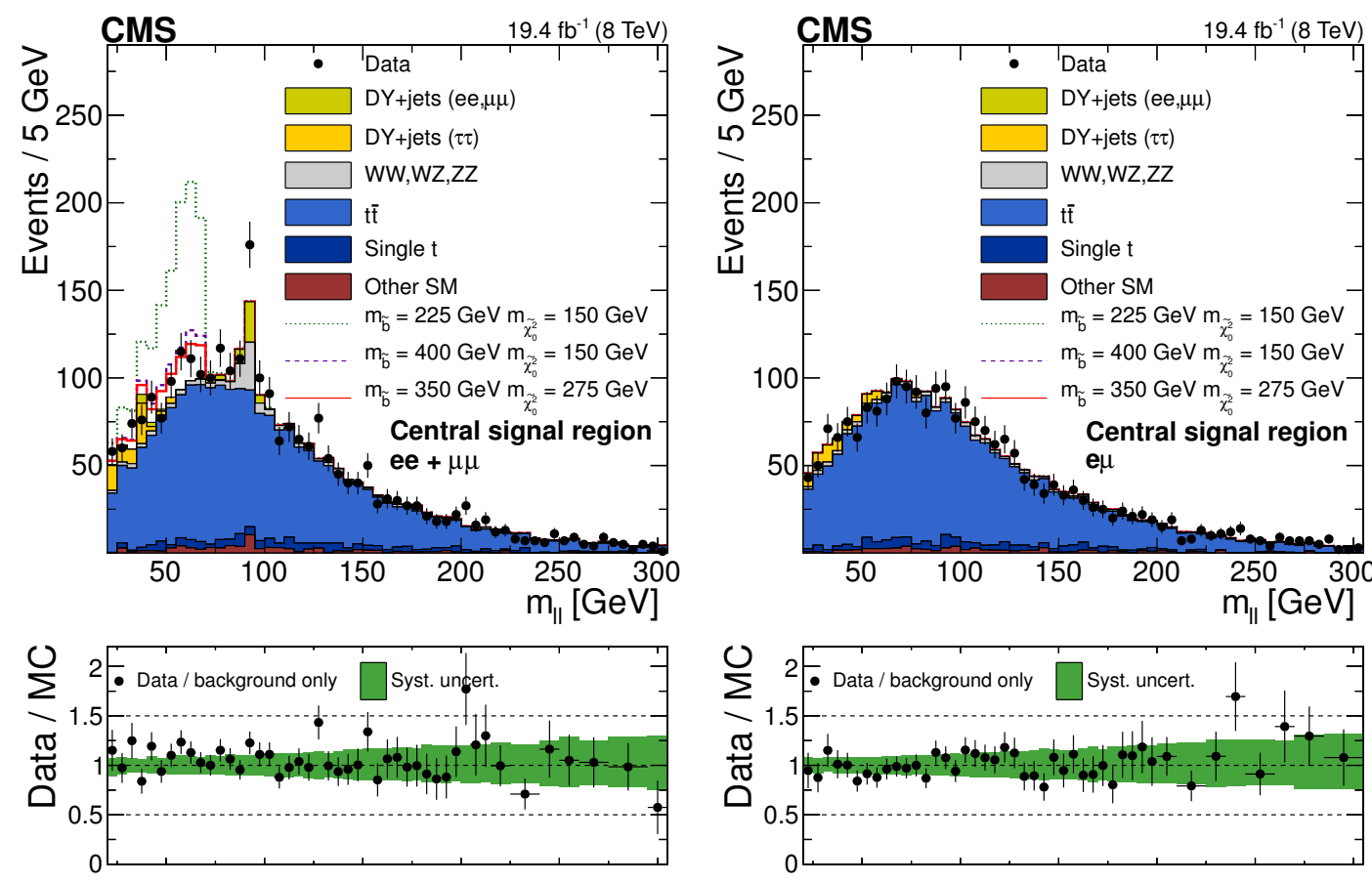

Figure 6. Data compared with SM simulation for the SF (left) and OF (right) event samples in the central region. Example signal scenarios based on the pair production of bottom squarks are shown (see text). In the ratio panel below each plot, the error bars on the points show the statistical uncertainties of the data and MC samples, while the shaded band indicates the MC systematic uncertainty.
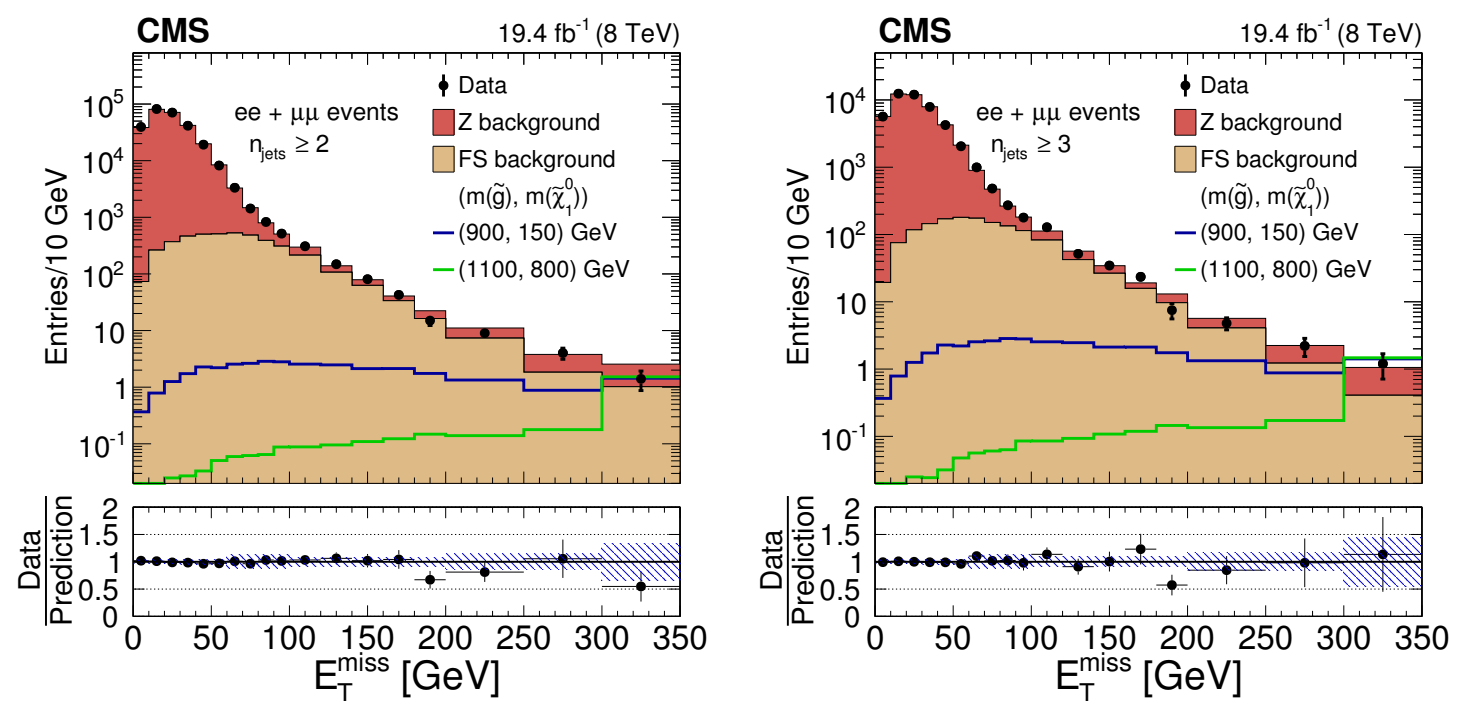

Figure 7. The $E_{\mathrm{T}}^{\text {miss }}$ distributions for the on-Z signal regions with (left) $\geq 2$ jets and (right) $\geq 3$ jets. The uncertainty band shown for the ratio includes both statistical and systematic uncertainties. Additionally, $E_{\mathrm{T}}^{\mathrm{miss}}$ distributions are drawn for two choices of masses in the GMSB scenario. The rightmost bins contain the overflow. 


\begin{tabular}{|l|c|c|c|}
\hline$E_{\mathrm{T}}^{\text {miss }}(\mathrm{GeV})$ & $100-200$ & $200-300$ & $>300$ \\
\hline DY background & $336 \pm 89$ & $28.6 \pm 8.6$ & $7.7 \pm 3.6$ \\
FS background & $868 \pm 57$ & $45.9 \pm 7.3$ & $5.1 \pm 2.3$ \\
\hline Total background & $1204 \pm 106$ & $74.5 \pm 11.3$ & $12.8 \pm 4.3$ \\
Data & 1187 & 65 & 7 \\
\hline \multicolumn{4}{|c|}{ GMSB signal yields } \\
\hline$m_{\widetilde{\mathrm{g}}}=900, m_{\widetilde{\chi}_{1}^{0}}=150$ & $22.1 \pm 0.4$ & $11.1 \pm 0.3$ & $7.2 \pm 0.2$ \\
$m_{\widetilde{\mathrm{g}}}=1100, m_{\widetilde{\chi}_{1}^{0}}=800$ & $1.1 \pm 0.04$ & $1.6 \pm 0.05$ & $7.6 \pm 0.1$ \\
\hline
\end{tabular}

Table 4. Event yields in the signal region for the dedicated on- $\mathrm{Z}$ counting experiment with $N_{\text {jets }} \geq 2$. Both statistical and systematic uncertainties are included for the background estimates. Signal yields are also shown for two choices of masses (in $\mathrm{GeV}$ ) in the GMSB scenario (statistical uncertainties only).

\begin{tabular}{|l|c|c|c|}
\hline$E_{\mathrm{T}}^{\mathrm{miss}}(\mathrm{GeV})$ & $100-200$ & $200-300$ & $>300$ \\
\hline DY background & $124 \pm 33$ & $12.7 \pm 3.8$ & $3.2 \pm 1.8$ \\
FS background & $354 \pm 28$ & $26.5 \pm 5.4$ & $2.0 \pm 1.4$ \\
\hline Total background & $478 \pm 43$ & $39.2 \pm 6.6$ & $5.3 \pm 2.3$ \\
Data & 490 & 35 & 6 \\
\hline \multicolumn{4}{|c|}{ GMSB signal yields } \\
\hline$m_{\widetilde{\mathrm{g}}}=900, m_{\widetilde{\chi}_{1}^{0}}=150$ & $22.0 \pm 0.4$ & $11.0 \pm 0.3$ & $7.1 \pm 0.2$ \\
$m_{\widetilde{\mathrm{g}}}=1100, m_{\widetilde{\chi}_{1}^{0}}=800$ & $1.1 \pm 0.04$ & $1.5 \pm 0.05$ & $7.4 \pm 0.1$ \\
\hline
\end{tabular}

Table 5. Event yields in the signal region for the dedicated on- $Z$ counting experiment with $N_{\text {jets }} \geq$ 3. Both statistical and systematic uncertainties are included for the background estimates. Signal yields are also shown for two choices of masses (in GeV) in the GMSB (statistical uncertainties only).

\section{Uncertainties in signal modeling}

Systematic uncertainties associated with the estimation of the SM background are discussed in section 5. This section describes uncertainties in the signal modeling. The impact of the uncertainties on the considered signal models is shown in table 6 .

The uncertainty related to the measurement of the integrated luminosity is $2.6 \%$ [36]. The uncertainty related to the parton distribution functions (PDF) is evaluated using the PDF4LHC recommendations [37-41] and amounts to $0-6 \%$ in the signal acceptance. Uncertainties in the modeling of the reconstruction, identification, and isolation efficiencies amount to $1 \%$ per lepton. The uncertainty of the corrections to account for lepton reconstruction differences between the fast and full simulations is $1 \%$ per lepton. The uncertainty in the trigger efficiencies is about $5 \%$. Uncertainties in the muon momentum scale are negligible, whereas for electrons the uncertainty in the energy scale is $0.6 \%(1.5 \%)$ in the barrel (endcap) region, leading to a $0-5 \%$ uncertainty in the signal yield. The uncertainty 


\begin{tabular}{|l|c|}
\hline Uncertainty source & Impact on signal yield [\%] \\
\hline Luminosity & 2.6 \\
PDFs on acceptance & $0-6$ \\
Lepton identification/isolation & 2 \\
Fast simulation lepton identification/isolation & 2 \\
Dilepton trigger & 5 \\
Lepton energy scale & $0-5$ \\
$E_{\mathrm{T}}^{\text {miss }}$ & $0-8$ \\
Jet energy scale/resolution & $0-8$ \\
ISR modeling & $0-14$ \\
Additional interactions & 1 \\
\hline
\end{tabular}

Table 6. Summary of systematic uncertainties for the signal efficiency.

in the jet energy scale [42] varies between $0-8 \%$. The uncertainty in the jet energy scale is propagated to evaluate the uncertainty associated with the $E_{\mathrm{T}}^{\mathrm{miss}}$ distribution, which is found to be $0-8 \%$. The uncertainty associated with the modeling of initial-state radiation (ISR) [43] is $0-14 \%$. The uncertainty in the correction to account for pileup in the simulation is evaluated by shifting the inelastic cross section by $\pm 5 \%$. The impact on the signal yield is found to be about $1 \%$.

\section{Interpretation}

Based on the results of the counting experiments, exclusion limits are determined. The kinematic fit is not used for this purpose because it assumes a specific shape for the dilepton mass spectrum of signal events and so is more model dependent. The limits are calculated at $95 \%$ confidence level (CL) using the $\mathrm{CL}_{\mathrm{s}}$ criterion with the LHC-style test statistic $[34,44,45]$, taking into account the statistical and systematic uncertainties in the signal yields and the background predictions discussed in sections 8 and 5, respectively. The different systematic uncertainties are considered to be uncorrelated with each other, but fully correlated among the different signal regions. The systematic uncertainties are treated as nuisance parameters and are parametrized with log-normal distributions.

For the fixed- and slepton-edge scenarios all six signal regions (low-mass, on-Z, and high-mass, for the central and forward lepton regions) are combined. The resulting exclusion limits for these scenarios are shown in figure 8. The production of bottom squarks is considered, as the excess observed in data consists predominantly of events with at least one $\mathrm{b}$ jet. In the fixed-edge scenario, the mass difference between the $\widetilde{\chi}_{2}^{0}$ and $\widetilde{\chi}_{1}^{0}$ neutralinos is fixed to $70 \mathrm{GeV}$, resulting in an edge in the $m_{\ell \ell}$ spectrum at this value. This choice for the edge position is motivated by the observed excess in the low-mass region of the counting experiment. Bottom-squark masses between 200 and $350 \mathrm{GeV}$ are probed, 

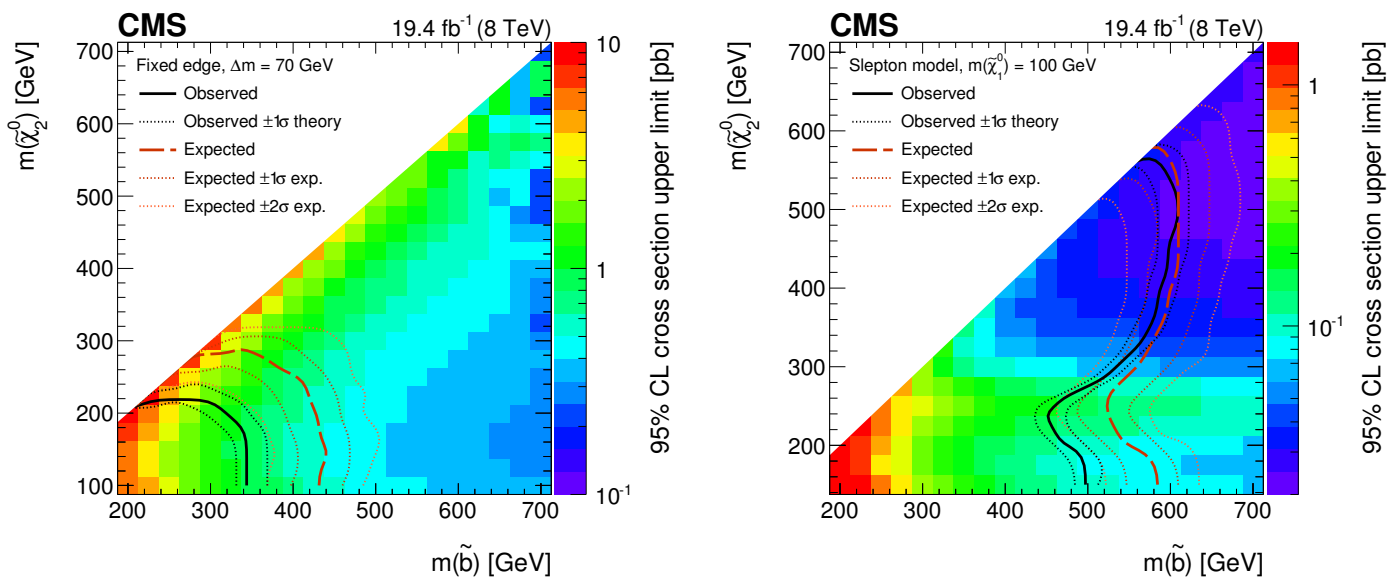

Figure 8. Exclusion limits at 95\% CL for the fixed- (left) and slepton-edge (right) scenarios in the $m_{\widetilde{\mathrm{b}}}-m_{\widetilde{\chi}_{2}^{0}}$ plane. The color indicates the excluded cross section for each considered point in parameter space. The intersections of the theoretical cross section with the expected and observed limits are indicated by the solid and hatched lines. The 1 standard deviation $(\sigma)$ experimental and theoretical uncertainty contours are shown as dotted lines.

depending on the value of the $\widetilde{\chi}_{2}^{0}$ mass. In the slepton-edge scenario, the $\widetilde{\chi}_{1}^{0}$ mass is set to $100 \mathrm{GeV}$, leaving the position of the edge as a free parameter that approximately corresponds to the mass difference between the $\widetilde{\chi}_{2}^{0}$ and $\widetilde{\chi}_{1}^{0}$. The branching fraction into dilepton final states is larger than in the fixed-edge scenario, and bottom-squark masses between 450 and $600 \mathrm{GeV}$ are probed, again depending on the value of the $\widetilde{\chi}_{2}^{0}$ mass. The loss of sensitivity seen in figure 8 (right) for $\widetilde{\chi}_{2}^{0}$ masses around $250 \mathrm{GeV}$ occurs because the peak of the triangular signal shape lies within, or close to, the gaps in acceptance between the low-mass and on-Z, or the on- $\mathrm{Z}$ and high-mass signal regions.

The results from the dedicated on-Z signal regions are used to place limits on the GMSB scenario. In this scenario, there are two free parameters: the masses of the gluino $\left(m_{\widetilde{\mathrm{g}}}\right)$ and the $\widetilde{\chi}_{1}^{0}\left(m_{\widetilde{\chi}_{1}^{0}}\right)$. As signal events typically have large jet multiplicities, the exclusive bins requiring $N_{\text {jets }} \geq 3$ and $E_{\mathrm{T}}^{\text {miss }}$ in the ranges $100<E_{\mathrm{T}}^{\text {miss }}<200 \mathrm{GeV}, 200<E_{\mathrm{T}}^{\text {miss }}<300 \mathrm{GeV}$, and $E_{\mathrm{T}}^{\text {miss }}>300 \mathrm{GeV}$ are used. The results are shown in figure 9 in the plane of $m_{\widetilde{\chi}_{1}^{0}}$ versus $m_{\widetilde{\mathrm{g}}}$. These results probe gluino masses up to about $900-1100 \mathrm{GeV}$ depending on the $\widetilde{\chi}_{1}^{0}$ mass. The limit is least stringent when $m_{\widetilde{\chi}_{1}^{0}}$ is close to the $\mathrm{Z}$ boson mass.

\section{Summary}

We have presented a search for physics beyond the standard model in the opposite-sign dilepton final state using a data sample of pp collisions collected at a center-of-mass energy of $8 \mathrm{TeV}$, corresponding to an integrated luminosity of $19.4 \mathrm{fb}^{-1}$, recorded with the CMS detector in 2012. Searches are performed for signals that either produce a kinematic edge, or a peak at the $\mathrm{Z}$ boson mass, in the dilepton invariant mass distribution. For regions dominated by flavor-symmetric backgrounds, i.e., backgrounds that produce opposite-flavor events $\left(\mathrm{e}^{+} \mu^{-}, \mathrm{e}^{-} \mu^{+}\right)$as often as same-flavor events $\left(\mathrm{e}^{+} \mathrm{e}^{-}, \mu^{+} \mu^{-}\right)$, we achieve a precision of about $5 \%(10 \%)$ for the estimated number of standard model background events in the 


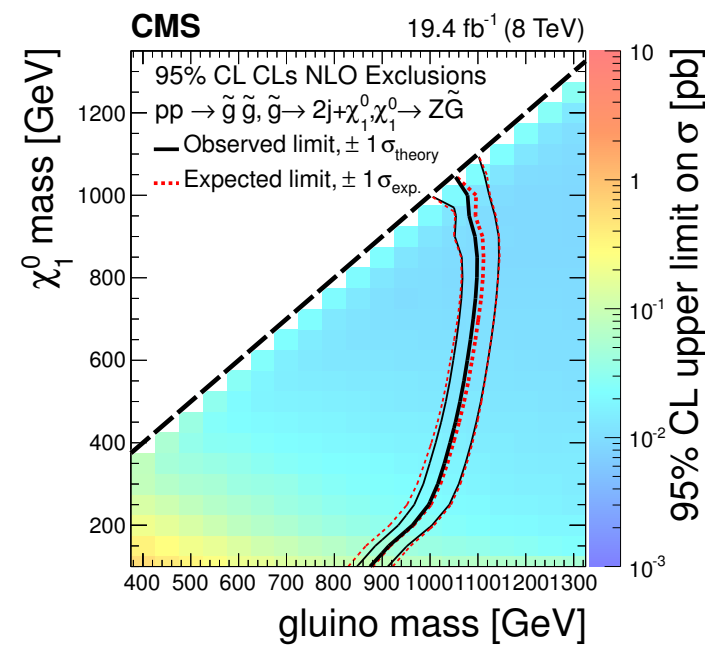

Figure 9. Exclusion limits at $95 \% \mathrm{CL}$ for the GMSB scenario in the $m_{\widetilde{\mathrm{g}}}-m_{\widetilde{\chi}_{1}^{0}}$ plane. The color indicates the excluded cross section for each considered point in parameter space. The intersections of the theoretical cross section with the expected and observed limits are indicated by the solid and hatched lines. The 1 standard deviation $(\sigma)$ experimental and theoretical uncertainty contours are shown as dotted lines.

central (forward) lepton rapidity regions. We do not observe evidence for a statistically significant signal. The maximum deviation from the null hypothesis is at the level of 2.6 standard deviations and is observed in the dilepton mass window $20<m_{\ell \ell}<70 \mathrm{GeV}$.

We interpret the results of the search for a kinematic edge in the context of simplified models consisting of bottom-squark pair production, with each bottom-squark $\widetilde{b}$ decaying to a bottom quark and the $\widetilde{\chi}_{2}^{0}$ neutralino. Exclusion limits are set in the $m_{\widetilde{\mathrm{b}}}-m_{\widetilde{\chi}_{2}^{0}}$ mass plane for two scenarios. In the fixed-edge scenario, the mass difference between the $\widetilde{\chi}_{2}^{0}$ and $\widetilde{\chi}_{1}^{0}$ particles is fixed to $70 \mathrm{GeV}$. Bottom-squark masses between 200 and $350 \mathrm{GeV}$ are excluded at $95 \%$ confidence level in this scenario. In the slepton-edge scenario, the $\widetilde{\chi}_{2}^{0}$ decays to an on- or off-shell Z boson and the $\widetilde{\chi}_{1}^{0}$ lightest supersymmetric particle or to a slepton and a lepton, with a $50 \%$ probability for each possibility. Bottom-squark masses between 450 and $600 \mathrm{GeV}$ are excluded at $95 \%$ confidence level in this second scenario. In both scenarios, the sensitivity depends on the mass of the $\widetilde{\chi}_{2}^{0}$. Finally, a dedicated search for events containing an on-shell $\mathrm{Z}$ boson is interpreted in a model of gauge-mediated supersymmetry breaking, in which the $\mathrm{Z}$ bosons are produced in decay chains initiated through gluino pair production. Gluino masses between 900 and $1100 \mathrm{GeV}$ are excluded at $95 \%$ confidence level, depending on the mass of the lightest neutralino $\widetilde{\chi}_{1}^{0}$.

\section{Acknowledgments}

We congratulate our colleagues in the CERN accelerator departments for the excellent performance of the LHC and thank the technical and administrative staffs at CERN and at other CMS institutes for their contributions to the success of the CMS effort. In addition, we gratefully acknowledge the computing centers and personnel of the Worldwide 
LHC Computing Grid for delivering so effectively the computing infrastructure essential to our analyses. Finally, we acknowledge the enduring support for the construction and operation of the LHC and the CMS detector provided by the following funding agencies: BMWFW and FWF (Austria); FNRS and FWO (Belgium); CNPq, CAPES, FAPERJ, and FAPESP (Brazil); MES (Bulgaria); CERN; CAS, MoST, and NSFC (China); COLCIENCIAS (Colombia); MSES and CSF (Croatia); RPF (Cyprus); MoER, ERC IUT and ERDF (Estonia); Academy of Finland, MEC, and HIP (Finland); CEA and CNRS/IN2P3 (France); BMBF, DFG, and HGF (Germany); GSRT (Greece); OTKA and NIH (Hungary); DAE and DST (India); IPM (Iran); SFI (Ireland); INFN (Italy); MSIP and NRF (Republic of Korea); LAS (Lithuania); MOE and UM (Malaysia); CINVESTAV, CONACYT, SEP, and UASLP-FAI (Mexico); MBIE (New Zealand); PAEC (Pakistan); MSHE and NSC (Poland); FCT (Portugal); JINR (Dubna); MON, RosAtom, RAS and RFBR (Russia); MESTD (Serbia); SEIDI and CPAN (Spain); Swiss Funding Agencies (Switzerland); MST (Taipei); ThEPCenter, IPST, STAR and NSTDA (Thailand); TUBITAK and TAEK (Turkey); NASU and SFFR (Ukraine); STFC (United Kingdom); DOE and NSF (U.S.A.).

Individuals have received support from the Marie-Curie program and the European Research Council and EPLANET (European Union); the Leventis Foundation; the A. P. Sloan Foundation; the Alexander von Humboldt Foundation; the Belgian Federal Science Policy Office; the Fonds pour la Formation à la Recherche dans l'Industrie et dans l'Agriculture (FRIA-Belgium); the Agentschap voor Innovatie door Wetenschap en Technologie (IWTBelgium); the Ministry of Education, Youth and Sports (MEYS) of the Czech Republic; the Council of Science and Industrial Research, India; the HOMING PLUS program of Foundation for Polish Science, cofinanced from European Union, Regional Development Fund; the Compagnia di San Paolo (Torino); the Consorzio per la Fisica (Trieste); MIUR project 20108T4XTM (Italy); the Thalis and Aristeia programs cofinanced by EU-ESF and the Greek NSRF; and the National Priorities Research Program by Qatar National Research Fund.

Open Access. This article is distributed under the terms of the Creative Commons Attribution License (CC-BY 4.0), which permits any use, distribution and reproduction in any medium, provided the original author(s) and source are credited.

\section{References}

[1] CMS collaboration, The CMS experiment at the CERN LHC, 2008 JINST 3 S08004 [INSPIRE].

[2] S.P. Martin, A supersymmetry primer, Adv. Ser. Direct. High Energy Phys. 21 (2010) 1 [hep-ph/9709356] [INSPIRE].

[3] I. Hinchliffe, F.E. Paige, M.D. Shapiro, J. Soderqvist and W. Yao, Precision SUSY measurements at CERN LHC, Phys. Rev. D 55 (1997) 5520 [hep-ph/9610544] [INSPIRE].

[4] CMS collaboration, Search for new physics in events with opposite-sign leptons, jets and missing transverse energy in pp collisions at $\sqrt{s}=7$ TeV, Phys. Lett. B 718 (2013) 815 [arXiv:1206.3949] [INSPIRE]. 
[5] CMS collaboration, Search for physics beyond the standard model in events with a $Z$ boson, jets and missing transverse energy in pp collisions at $\sqrt{s}=7$ TeV, Phys. Lett. B 716 (2012) 260 [arXiv: 1204.3774] [INSPIRE].

[6] ATLAS collaboration, Search for direct production of charginos, neutralinos and sleptons in final states with two leptons and missing transverse momentum in pp collisions at $\sqrt{s}=8 \mathrm{TeV}$ with the ATLAS detector, JHEP 05 (2014) 071 [arXiv:1403.5294] [INSPIRE].

[7] ATLAS collaboration, Search for direct top-squark pair production in final states with two leptons in pp collisions at $\sqrt{s}=8$ TeV with the ATLAS detector, JHEP 06 (2014) 124 [arXiv: 1403.4853] [INSPIRE].

[8] LHC New Physics Working Group, D. Alves et al., Simplified models for LHC new physics searches, J. Phys. G 39 (2012) 105005 [arXiv:1105.2838] [InSPIRE].

[9] K.T. Matchev and S.D. Thomas, Higgs and $Z$ boson signatures of supersymmetry, Phys. Rev. D 62 (2000) 077702 [hep-ph/9908482] [INSPIRE].

[10] P. Meade, M. Reece and D. Shih, Prompt decays of general neutralino NLSPs at the Tevatron, JHEP 05 (2010) 105 [arXiv:0911.4130] [INSPIRE].

[11] J.T. Ruderman and D. Shih, General neutralino NLSPs at the early LHC, JHEP 08 (2012) 159 [arXiv: 1103.6083] [INSPIRE].

[12] J. Alwall, M. Herquet, F. Maltoni, O. Mattelaer and T. Stelzer, MadGraph 5: going beyond, JHEP 06 (2011) 128 [arXiv: 1106.0522] [INSPIRE].

[13] J. Alwall et al., The automated computation of tree-level and next-to-leading order differential cross sections and their matching to parton shower simulations, JHEP 07 (2014) 079 [arXiv: 1405.0301] [INSPIRE].

[14] T. Sjöstrand, S. Mrenna and P.Z. Skands, PYTHIA 6.4 physics and manual, JHEP 05 (2006) 026 [hep-ph/0603175] [INSPIRE].

[15] S. Abdullin et al., The fast simulation of the CMS detector at LHC, J. Phys. Conf. Ser. 331 (2012) 032049.

[16] GEANT4 collaboration, S. Agostinelli et al., GEANT4 - a simulation toolkit, Nucl. Instrum. Meth. A 506 (2003) 250 [InSPIRE].

[17] W. Beenakker, R. Hopker, M. Spira and P.M. Zerwas, Squark and gluino production at hadron colliders, Nucl. Phys. B 492 (1997) 51 [hep-ph/9610490] [INSPIRE].

[18] A. Kulesza and L. Motyka, Threshold resummation for squark-antisquark and gluino-pair production at the LHC, Phys. Rev. Lett. 102 (2009) 111802 [arXiv:0807.2405] [InSPIRE].

[19] A. Kulesza and L. Motyka, Soft gluon resummation for the production of gluino-gluino and squark-antisquark pairs at the LHC, Phys. Rev. D 80 (2009) 095004 [arXiv:0905.4749] [INSPIRE].

[20] W. Beenakker et al., Soft-gluon resummation for squark and gluino hadroproduction, JHEP 12 (2009) 041 [arXiv: 0909.4418] [INSPIRE].

[21] W. Beenakker et al., Squark and gluino hadroproduction, Int. J. Mod. Phys. A 26 (2011) 2637 [arXiv: 1105.1110] [INSPIRE].

[22] M. Krämer et al., Supersymmetry production cross sections in pp collisions at $\sqrt{s}=7$ TeV,

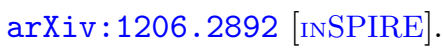

[23] CMS collaboration, Performance of electron reconstruction and selection with the CMS detector in proton-proton collisions at $\sqrt{s}=8 \mathrm{TeV}$, arXiv:1502.02701 [INSPIRE]. 
[24] CMS collaboration, Performance of CMS muon reconstruction in pp collision events at $\sqrt{s}=7 \mathrm{TeV}, 2012 \operatorname{JINST} 7 \mathrm{P} 10002$ [arXiv:1206.4071] [INSPIRE].

[25] M. Cacciari, G.P. Salam and G. Soyez, FastJet user manual, Eur. Phys. J. C 72 (2012) 1896 [arXiv: 1111.6097] [INSPIRE].

[26] CMS collaboration, Performance of $\tau$-lepton reconstruction and identification in CMS, 2012 JINST 7 P01001 [arXiv:1109.6034] [INSPIRE].

[27] M. Cacciari, G.P. Salam and G. Soyez, The anti- $k_{t}$ jet clustering algorithm, JHEP 04 (2008) 063 [arXiv:0802.1189] [INSPIRE].

[28] M. Cacciari and G.P. Salam, Dispelling the $N^{3}$ myth for the $k_{t}$ jet-finder, Phys. Lett. B 641 (2006) 57 [hep-ph/0512210] [INSPIRE].

[29] M. Cacciari and G.P. Salam, Pileup subtraction using jet areas, Phys. Lett. B 659 (2008) 119 [arXiv:0707.1378] [INSPIRE].

[30] Y. Li and F. Petriello, Combining QCD and electroweak corrections to dilepton production in FEWZ, Phys. Rev. D 86 (2012) 094034 [arXiv: 1208.5967] [InSPIRE].

[31] Particle Data Group, K.A. Olive et al., Review of particle physics, Chin. Phys. C 38 (2014) 090001.

[32] M.J. Oreglia, A study of the reactions $\psi^{\prime} \rightarrow \gamma \gamma \psi$, Ph.D. thesis, Stanford University, Stanford, U.S.A. (1980), see appendix D [SLAC-R-236].

[33] F. James and M. Roos, Minuit: a system for function minimization and analysis of the parameter errors and correlations, Comput. Phys. Commun. 10 (1975) 343 [INSPIRE].

[34] ATLAS collaboration, Procedure for the LHC Higgs boson search combination in summer 2011, ATL-PHYS-PUB-2011-011 (2011).

[35] CMS collaboration, Identification of b-quark jets with the CMS experiment, 2013 JINST 8 P04013 [arXiv: 1211.4462] [INSPIRE].

[36] CMS Collaboration, CMS luminosity based on pixel cluster counting — Summer 2013 Update, CMS-PAS-LUM-13-001 (2013).

[37] S. Alekhin et al., The PDF4LHC working group interim report, arXiv: 1101.0536 [INSPIRE].

[38] M. Botje et al., The PDF 4 LHC working group interim recommendations, arXiv: 1101.0538 [INSPIRE].

[39] R.D. Ball et al., Parton distributions with LHC data, Nucl. Phys. B 867 (2013) 244 [arXiv: 1207.1303] [INSPIRE].

[40] A.D. Martin, W.J. Stirling, R.S. Thorne and G. Watt, Parton distributions for the LHC, Eur. Phys. J. C 63 (2009) 189 [arXiv:0901.0002] [InSPIRE].

[41] H.-L. Lai et al., New parton distributions for collider physics, Phys. Rev. D 82 (2010) 074024 [arXiv: 1007.2241] [INSPIRE].

[42] CMS collaboration, Determination of jet energy calibration and transverse momentum resolution in CMS, 2011 JINST $6 \mathrm{P} 11002$.

[43] CMS collaboration, Search for top-squark pair production in the single-lepton final state in pp collisions at $\sqrt{s}=8 \mathrm{TeV}$, Eur. Phys. J. C 73 (2013) 2677 [arXiv:1308.1586] [InSPIRE].

[44] T. Junk, Confidence level computation for combining searches with small statistics, Nucl. Instrum. Meth. A 434 (1999) 435 [hep-ex/9902006] [INSPIRE].

[45] A.L. Read, Presentation of search results: the CL(s) technique, J. Phys. G 28 (2002) 2693 [INSPIRE]. 


\section{The CMS collaboration}

\section{Yerevan Physics Institute, Yerevan, Armenia}

V. Khachatryan, A.M. Sirunyan, A. Tumasyan

\section{Institut für Hochenergiephysik der OeAW, Wien, Austria}

W. Adam, T. Bergauer, M. Dragicevic, J. Erö, M. Friedl, R. Frühwirth ${ }^{1}$, V.M. Ghete, C. Hartl, N. Hörmann, J. Hrubec, M. Jeitler ${ }^{1}$, W. Kiesenhofer, V. Knünz, M. Krammer ${ }^{1}$, I. Krätschmer, D. Liko, I. Mikulec, D. Rabady², B. Rahbaran, H. Rohringer, R. Schöfbeck, J. Strauss, W. Treberer-Treberspurg, W. Waltenberger, C.-E. Wulz ${ }^{1}$

National Centre for Particle and High Energy Physics, Minsk, Belarus

V. Mossolov, N. Shumeiko, J. Suarez Gonzalez

Universiteit Antwerpen, Antwerpen, Belgium

S. Alderweireldt, S. Bansal, T. Cornelis, E.A. De Wolf, X. Janssen, A. Knutsson, J. Lauwers, S. Luyckx, S. Ochesanu, R. Rougny, M. Van De Klundert, H. Van Haevermaet, P. Van Mechelen, N. Van Remortel, A. Van Spilbeeck

Vrije Universiteit Brussel, Brussel, Belgium

F. Blekman, S. Blyweert, J. D'Hondt, N. Daci, N. Heracleous, J. Keaveney, S. Lowette, M. Maes, A. Olbrechts, Q. Python, D. Strom, S. Tavernier, W. Van Doninck, P. Van Mulders, G.P. Van Onsem, I. Villella

Université Libre de Bruxelles, Bruxelles, Belgium

C. Caillol, B. Clerbaux, G. De Lentdecker, D. Dobur, L. Favart, A.P.R. Gay, A. Grebenyuk, A. Léonard, A. Mohammadi, L. Perniè ${ }^{2}$, A. Randle-conde, T. Reis, T. Seva, L. Thomas, C. Vander Velde, P. Vanlaer, J. Wang, F. Zenoni

\section{Ghent University, Ghent, Belgium}

V. Adler, K. Beernaert, L. Benucci, A. Cimmino, S. Costantini, S. Crucy, A. Fagot, G. Garcia, J. Mccartin, A.A. Ocampo Rios, D. Poyraz, D. Ryckbosch, S. Salva Diblen, M. Sigamani, N. Strobbe, F. Thyssen, M. Tytgat, E. Yazgan, N. Zaganidis

\section{Université Catholique de Louvain, Louvain-la-Neuve, Belgium}

S. Basegmez, C. Beluffi ${ }^{3}$, G. Bruno, R. Castello, A. Caudron, L. Ceard, G.G. Da Silveira, C. Delaere, T. du Pree, D. Favart, L. Forthomme, A. Giammanco ${ }^{4}$, J. Hollar, A. Jafari, P. Jez, M. Komm, V. Lemaitre, C. Nuttens, D. Pagano, L. Perrini, A. Pin, K. Piotrzkowski, A. Popov ${ }^{5}$, L. Quertenmont, M. Selvaggi, M. Vidal Marono, J.M. Vizan Garcia

\section{Université de Mons, Mons, Belgium}

N. Beliy, T. Caebergs, E. Daubie, G.H. Hammad

Centro Brasileiro de Pesquisas Fisicas, Rio de Janeiro, Brazil

W.L. Aldá Júnior, G.A. Alves, L. Brito, M. Correa Martins Junior, T. Dos Reis Martins, J. Molina, C. Mora Herrera, M.E. Pol, P. Rebello Teles 
Universidade do Estado do Rio de Janeiro, Rio de Janeiro, Brazil

W. Carvalho, J. Chinellato ${ }^{6}$, A. Custódio, E.M. Da Costa, D. De Jesus Damiao, C. De Oliveira Martins, S. Fonseca De Souza, H. Malbouisson, D. Matos Figueiredo, L. Mundim, H. Nogima, W.L. Prado Da Silva, J. Santaolalla, A. Santoro, A. Sznajder, E.J. Tonelli Manganote $^{6}$, A. Vilela Pereira

Universidade Estadual Paulista $^{a}$, Universidade Federal do $\mathrm{ABC}^{b}$, São Paulo, Brazil

C.A. Bernardes ${ }^{b}$, S. Dogra ${ }^{a}$, T.R. Fernandez Perez Tomei ${ }^{a}$, E.M. Gregores ${ }^{b}$, P.G. Mercadante ${ }^{b}$, S.F. Novaes ${ }^{a}$, Sandra S. Padula ${ }^{a}$

Institute for Nuclear Research and Nuclear Energy, Sofia, Bulgaria

A. Aleksandrov, V. Genchev², R. Hadjiiska, P. Iaydjiev, A. Marinov, S. Piperov, M. Rodozov, S. Stoykova, G. Sultanov, M. Vutova

University of Sofia, Sofia, Bulgaria

A. Dimitrov, I. Glushkov, L. Litov, B. Pavlov, P. Petkov

Institute of High Energy Physics, Beijing, China

J.G. Bian, G.M. Chen, H.S. Chen, M. Chen, T. Cheng, R. Du, C.H. Jiang, R. Plestina ${ }^{7}$, F. Romeo, J. Tao, Z. Wang

State Key Laboratory of Nuclear Physics and Technology, Peking University, Beijing, China

C. Asawatangtrakuldee, Y. Ban, S. Liu, Y. Mao, S.J. Qian, D. Wang, Z. Xu, F. Zhang ${ }^{8}$,

L. Zhang, W. Zou

Universidad de Los Andes, Bogota, Colombia

C. Avila, A. Cabrera, L.F. Chaparro Sierra, C. Florez, J.P. Gomez, B. Gomez Moreno, J.C. Sanabria

University of Split, Faculty of Electrical Engineering, Mechanical Engineering and Naval Architecture, Split, Croatia

N. Godinovic, D. Lelas, D. Polic, I. Puljak

University of Split, Faculty of Science, Split, Croatia

Z. Antunovic, M. Kovac

Institute Rudjer Boskovic, Zagreb, Croatia

V. Brigljevic, K. Kadija, J. Luetic, D. Mekterovic, L. Sudic

University of Cyprus, Nicosia, Cyprus

A. Attikis, G. Mavromanolakis, J. Mousa, C. Nicolaou, F. Ptochos, P.A. Razis, H. Rykaczewski

Charles University, Prague, Czech Republic

M. Bodlak, M. Finger, M. Finger Jr. ${ }^{9}$ 
Academy of Scientific Research and Technology of the Arab Republic of Egypt, Egyptian Network of High Energy Physics, Cairo, Egypt

Y. Assran $^{10}$, A. Ellithi Kamel ${ }^{11}$, M.A. Mahmoud ${ }^{12}$, A. Radi ${ }^{13,14}$

National Institute of Chemical Physics and Biophysics, Tallinn, Estonia

M. Kadastik, M. Murumaa, M. Raidal, A. Tiko

Department of Physics, University of Helsinki, Helsinki, Finland

P. Eerola, M. Voutilainen

Helsinki Institute of Physics, Helsinki, Finland

J. Härkönen, V. Karimäki, R. Kinnunen, M.J. Kortelainen, T. Lampén, K. Lassila-Perini,

S. Lehti, T. Lindén, P. Luukka, T. Mäenpää, T. Peltola, E. Tuominen, J. Tuominiemi,

E. Tuovinen, L. Wendland

Lappeenranta University of Technology, Lappeenranta, Finland

J. Talvitie, T. Tuuva

DSM/IRFU, CEA/Saclay, Gif-sur-Yvette, France

M. Besancon, F. Couderc, M. Dejardin, D. Denegri, B. Fabbro, J.L. Faure, C. Favaro, F. Ferri, S. Ganjour, A. Givernaud, P. Gras, G. Hamel de Monchenault, P. Jarry, E. Locci, J. Malcles, J. Rander, A. Rosowsky, M. Titov

Laboratoire Leprince-Ringuet, Ecole Polytechnique, IN2P3-CNRS, Palaiseau, France

S. Baffioni, F. Beaudette, P. Busson, E. Chapon, C. Charlot, T. Dahms, L. Dobrzynski, N. Filipovic, A. Florent, R. Granier de Cassagnac, L. Mastrolorenzo, P. Miné, I.N. Naranjo, M. Nguyen, C. Ochando, G. Ortona, P. Paganini, S. Regnard, R. Salerno, J.B. Sauvan, Y. Sirois, C. Veelken, Y. Yilmaz, A. Zabi

Institut Pluridisciplinaire Hubert Curien, Université de Strasbourg, Université de Haute Alsace Mulhouse, CNRS/IN2P3, Strasbourg, France

J.-L. Agram ${ }^{15}$, J. Andrea, A. Aubin, D. Bloch, J.-M. Brom, E.C. Chabert, C. Collard, E. Conte ${ }^{15}$, J.-C. Fontaine ${ }^{15}$, D. Gelé, U. Goerlach, C. Goetzmann, A.-C. Le Bihan, K. Skovpen, P. Van Hove

Centre de Calcul de l'Institut National de Physique Nucleaire et de Physique des Particules, CNRS/IN2P3, Villeurbanne, France

S. Gadrat

Université de Lyon, Université Claude Bernard Lyon 1, CNRS-IN2P3, Institut de Physique Nucléaire de Lyon, Villeurbanne, France

S. Beauceron, N. Beaupere, C. Bernet ${ }^{7}$, G. Boudoul ${ }^{2}$, E. Bouvier, S. Brochet, C.A. Carrillo Montoya, J. Chasserat, R. Chierici, D. Contardo ${ }^{2}$, B. Courbon, P. Depasse, H. El Mamouni, J. Fan, J. Fay, S. Gascon, M. Gouzevitch, B. Ille, T. Kurca, M. Lethuillier, L. Mirabito, A.L. Pequegnot, S. Perries, J.D. Ruiz Alvarez, D. Sabes, L. Sgandurra, V. Sordini, M. Vander Donckt, P. Verdier, S. Viret, H. Xiao 
Institute of High Energy Physics and Informatization, Tbilisi State University, Tbilisi, Georgia

Z. Tsamalaidze ${ }^{9}$

\section{RWTH Aachen University, I. Physikalisches Institut, Aachen, Germany}

C. Autermann, S. Beranek, M. Bontenackels, M. Edelhoff, L. Feld, A. Heister, K. Klein, M. Lipinski, A. Ostapchuk, M. Preuten, F. Raupach, J. Sammet, S. Schael, C. Schomakers, J.F. Schulte, D. Sprenger, H. Weber, B. Wittmer, V. Zhukov ${ }^{5}$

RWTH Aachen University, III. Physikalisches Institut A, Aachen, Germany M. Ata, M. Brodski, E. Dietz-Laursonn, D. Duchardt, M. Erdmann, R. Fischer, A. Güth, T. Hebbeker, C. Heidemann, K. Hoepfner, D. Klingebiel, S. Knutzen, P. Kreuzer, M. Merschmeyer, A. Meyer, P. Millet, M. Olschewski, K. Padeken, P. Papacz, H. Reithler, S.A. Schmitz, L. Sonnenschein, D. Teyssier, S. Thüer

\section{RWTH Aachen University, III. Physikalisches Institut B, Aachen, Germany}

V. Cherepanov, Y. Erdogan, G. Flügge, H. Geenen, M. Geisler, W. Haj Ahmad, F. Hoehle, B. Kargoll, T. Kress, Y. Kuessel, A. Künsken, J. Lingemann ${ }^{2}$, A. Nowack, I.M. Nugent, C. Pistone, O. Pooth, A. Stahl

\section{Deutsches Elektronen-Synchrotron, Hamburg, Germany}

M. Aldaya Martin, I. Asin, N. Bartosik, J. Behr, U. Behrens, A.J. Bell, A. Bethani, K. Borras, A. Burgmeier, A. Cakir, L. Calligaris, A. Campbell, S. Choudhury, F. Costanza, C. Diez Pardos, G. Dolinska, S. Dooling, T. Dorland, G. Eckerlin, D. Eckstein, T. Eichhorn, G. Flucke, J. Garay Garcia, A. Geiser, A. Gizhko, P. Gunnellini, J. Hauk, M. Hempel ${ }^{16}$, H. Jung, A. Kalogeropoulos, O. Karacheban ${ }^{16}$, M. Kasemann, P. Katsas, J. Kieseler, C. Kleinwort, I. Korol, D. Krücker, W. Lange, J. Leonard, K. Lipka, A. Lobanov, W. Lohmann ${ }^{16}$, B. Lutz, R. Mankel, I. Marfin ${ }^{16}$, I.-A. Melzer-Pellmann, A.B. Meyer, G. Mittag, J. Mnich, A. Mussgiller, S. Naumann-Emme, A. Nayak, E. Ntomari, H. Perrey, D. Pitzl, R. Placakyte, A. Raspereza, P.M. Ribeiro Cipriano, B. Roland, E. Ron, M.Ö. Sahin, J. Salfeld-Nebgen, P. Saxena, T. Schoerner-Sadenius, M. Schröder, C. Seitz, S. Spannagel, A.D.R. Vargas Trevino, R. Walsh, C. Wissing

\section{University of Hamburg, Hamburg, Germany}

V. Blobel, M. Centis Vignali, A.R. Draeger, J. Erfle, E. Garutti, K. Goebel, M. Görner, J. Haller, M. Hoffmann, R.S. Höing, A. Junkes, H. Kirschenmann, R. Klanner, R. Kogler, T. Lapsien, T. Lenz, I. Marchesini, D. Marconi, J. Ott, T. Peiffer, A. Perieanu, N. Pietsch, J. Poehlsen, T. Poehlsen, D. Rathjens, C. Sander, H. Schettler, P. Schleper, E. Schlieckau, A. Schmidt, M. Seidel, V. Sola, H. Stadie, G. Steinbrück, D. Troendle, E. Usai, L. Vanelderen, A. Vanhoefer

\section{Institut für Experimentelle Kernphysik, Karlsruhe, Germany}

C. Barth, C. Baus, J. Berger, C. Böser, E. Butz, T. Chwalek, W. De Boer, A. Descroix, A. Dierlamm, M. Feindt, F. Frensch, M. Giffels, A. Gilbert, F. Hartmann², T. Hauth, U. Husemann, I. Katkov ${ }^{5}$, A. Kornmayer ${ }^{2}$, P. Lobelle Pardo, M.U. Mozer, T. Müller, 
Th. Müller, A. Nürnberg, G. Quast, K. Rabbertz, S. Röcker, H.J. Simonis, F.M. Stober, R. Ulrich, J. Wagner-Kuhr, S. Wayand, T. Weiler, R. Wolf

Institute of Nuclear and Particle Physics (INPP), NCSR Demokritos, Aghia Paraskevi, Greece

G. Anagnostou, G. Daskalakis, T. Geralis, V.A. Giakoumopoulou, A. Kyriakis, D. Loukas, A. Markou, C. Markou, A. Psallidas, I. Topsis-Giotis

University of Athens, Athens, Greece

A. Agapitos, S. Kesisoglou, A. Panagiotou, N. Saoulidou, E. Stiliaris, E. Tziaferi

University of Ioánnina, Ioánnina, Greece

X. Aslanoglou, I. Evangelou, G. Flouris, C. Foudas, P. Kokkas, N. Manthos, I. Papadopoulos, E. Paradas, J. Strologas

Wigner Research Centre for Physics, Budapest, Hungary

G. Bencze, C. Hajdu, P. Hidas, D. Horvath ${ }^{17}$, F. Sikler, V. Veszpremi, G. Vesztergombi ${ }^{18}$, A.J. Zsigmond

Institute of Nuclear Research ATOMKI, Debrecen, Hungary

N. Beni, S. Czellar, J. Karancsi ${ }^{19}$, J. Molnar, J. Palinkas, Z. Szillasi

University of Debrecen, Debrecen, Hungary

A. Makovec, P. Raics, Z.L. Trocsanyi, B. Ujvari

National Institute of Science Education and Research, Bhubaneswar, India

S.K. Swain

Panjab University, Chandigarh, India

S.B. Beri, V. Bhatnagar, R. Gupta, U.Bhawandeep, A.K. Kalsi, M. Kaur, R. Kumar, M. Mittal, N. Nishu, J.B. Singh

University of Delhi, Delhi, India

Ashok Kumar, Arun Kumar, S. Ahuja, A. Bhardwaj, B.C. Choudhary, A. Kumar, S. Malhotra, M. Naimuddin, K. Ranjan, V. Sharma

Saha Institute of Nuclear Physics, Kolkata, India

S. Banerjee, S. Bhattacharya, K. Chatterjee, S. Dutta, B. Gomber, Sa. Jain, Sh. Jain, R. Khurana, A. Modak, S. Mukherjee, D. Roy, S. Sarkar, M. Sharan

Bhabha Atomic Research Centre, Mumbai, India

A. Abdulsalam, D. Dutta, V. Kumar, A.K. Mohanty², L.M. Pant, P. Shukla, A. Topkar

Tata Institute of Fundamental Research, Mumbai, India

T. Aziz, S. Banerjee, S. Bhowmik ${ }^{20}$, R.M. Chatterjee, R.K. Dewanjee, S. Dugad, S. Ganguly, S. Ghosh, M. Guchait, A. Gurtu ${ }^{21}$, G. Kole, S. Kumar, M. Maity ${ }^{20}$, G. Majumder, K. Mazumdar, G.B. Mohanty, B. Parida, K. Sudhakar, N. Wickramage ${ }^{22}$

Indian Institute of Science Education and Research (IISER), Pune, India S. Sharma 
Institute for Research in Fundamental Sciences (IPM), Tehran, Iran

H. Bakhshiansohi, H. Behnamian, S.M. Etesami ${ }^{23}$, A. Fahim ${ }^{24}$, R. Goldouzian, M. Khakzad, M. Mohammadi Najafabadi, M. Naseri, S. Paktinat Mehdiabadi, F. Rezaei Hosseinabadi, B. Safarzadeh ${ }^{25}$, M. Zeinali

University College Dublin, Dublin, Ireland

M. Felcini, M. Grunewald

INFN Sezione di Bari ${ }^{a}$, Università di Bari ${ }^{b}$, Politecnico di Bari ${ }^{c}$, Bari, Italy

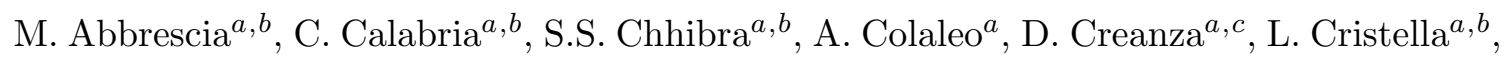

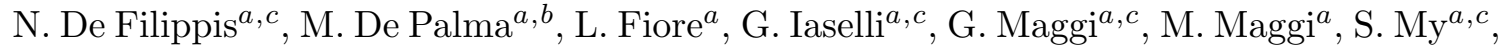
S. Nuzzo ${ }^{a, b}$, A. Pompili ${ }^{a, b}$, G. Pugliese ${ }^{a, c}$, R. Radogna ${ }^{a, b, 2}$, G. Selvaggi ${ }^{a, b}$, A. Sharma ${ }^{a}$, L. Silvestris ${ }^{a, 2}$, R. Venditti ${ }^{a}, b$, P. Verwilligen ${ }^{a}$

INFN Sezione di Bologna ${ }^{a}$, Università di Bologna ${ }^{b}$, Bologna, Italy

G. Abbiendi ${ }^{a}$, A.C. Benvenuti ${ }^{a}$, D. Bonacorsi ${ }^{a, b}$, S. Braibant-Giacomelli ${ }^{a, b}$, L. Brigliadoria ${ }^{a, b}$, R. Campanini ${ }^{a, b}$, P. Capiluppi ${ }^{a, b}$, A. Castro ${ }^{a, b}$, F.R. Cavallo ${ }^{a}$,

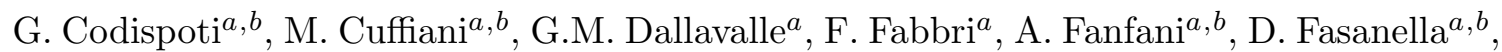
P. Giacomelli ${ }^{a}$, C. Grandi ${ }^{a}$, L. Guiducci ${ }^{a}, b$, S. Marcellini ${ }^{a}$, G. Masetti ${ }^{a}$, A. Montanari ${ }^{a}$,

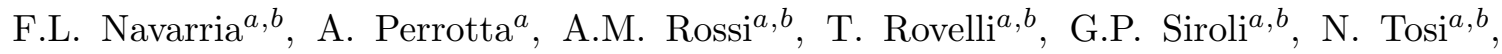
R. Travaglini ${ }^{a, b}$

INFN Sezione di Catania ${ }^{a}$, Università di Catania ${ }^{b}, \operatorname{CSFNSM}^{c}$, Catania, Italy

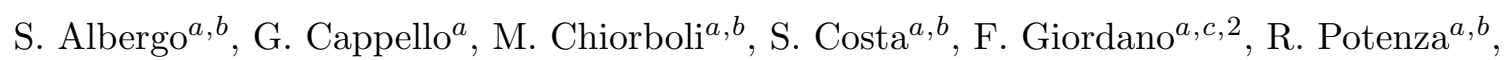
A. Tricomi ${ }^{a, b}$, C. Tuve ${ }^{a, b}$

INFN Sezione di Firenze ${ }^{a}$, Università di Firenze ${ }^{b}$, Firenze, Italy

G. Barbagli ${ }^{a}$, V. Ciulli ${ }^{a, b}$, C. Civinini ${ }^{a}$, R. D’Alessandro ${ }^{a, b}$, E. Focardi ${ }^{a, b}$, E. Gallo ${ }^{a}$, S. Gonzi ${ }^{a, b}$, V. Gori ${ }^{a, b}$, P. Lenzi ${ }^{a, b}$, M. Meschini ${ }^{a}$, S. Paoletti ${ }^{a}$, G. Sguazzoni ${ }^{a}$, A. Tropiano ${ }^{a, b}$

INFN Laboratori Nazionali di Frascati, Frascati, Italy

L. Benussi, S. Bianco, F. Fabbri, D. Piccolo

INFN Sezione di Genova ${ }^{a}$, Università di Genova ${ }^{b}$, Genova, Italy

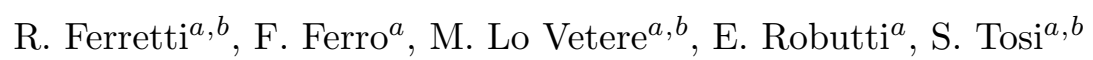

INFN Sezione di Milano-Bicocca ${ }^{a}$, Università di Milano-Bicocca ${ }^{b}$, Milano, Italy

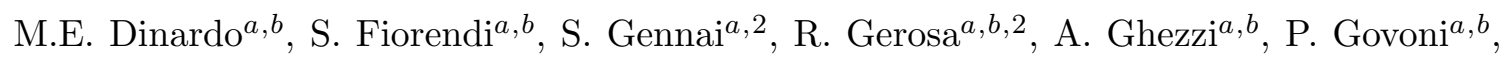
M.T. Lucchini ${ }^{a, b, 2}$, S. Malvezzi ${ }^{a}$, R.A. Manzoni ${ }^{a, b}$, A. Martelli ${ }^{a, b}$, B. Marzocchi ${ }^{a}, b, 2$,

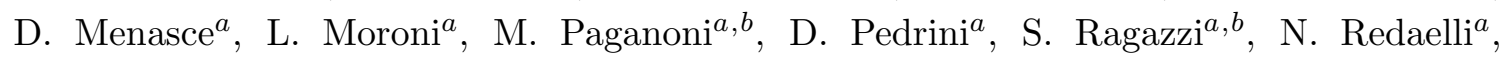
T. Tabarelli de Fatis ${ }^{a, b}$ 
INFN Sezione di Napoli ${ }^{a}$, Università di Napoli 'Federico II' ${ }^{b}$, Napoli, Italy, Università della Basilicata ${ }^{c}$, Potenza, Italy, Università G. Marconi ${ }^{d}$, Roma, Italy

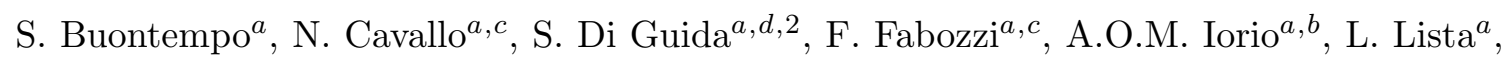
S. Meola ${ }^{a, d, 2}$, M. Merola ${ }^{a}$, P. Paolucci ${ }^{a, 2}$

INFN Sezione di Padova ${ }^{a}$, Università di Padova ${ }^{b}$, Padova, Italy, Università di Trento ${ }^{c}$, Trento, Italy

P. Azzi ${ }^{a}$, N. Bacchetta ${ }^{a}$, D. Bisello ${ }^{a, b}$, R. Carlin ${ }^{a, b}$, P. Checchia ${ }^{a}$, M. Dall'Osso ${ }^{a, b}$,

T. Dorigo ${ }^{a}$, U. Dosselli ${ }^{a}$, F. Fanzago ${ }^{a}$, F. Gasparini ${ }^{a, b}$, U. Gasparini ${ }^{a}, b$, F. Gonella ${ }^{a}$,

A. Gozzelino ${ }^{a}$, S. Lacaprara ${ }^{a}$, M. Margoni ${ }^{a}, b$ A.T. Meneguzzo ${ }^{a, b}$, J. Pazzini ${ }^{a, b}$,

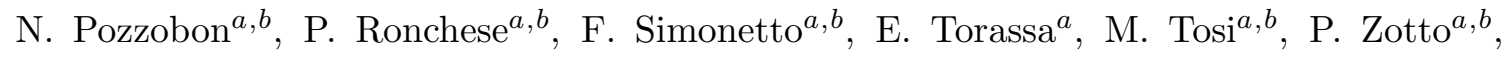

A. Zucchetta ${ }^{a, b}$, G. Zumerle ${ }^{a, b}$

INFN Sezione di Pavia ${ }^{a}$, Università di Pavia ${ }^{b}$, Pavia, Italy

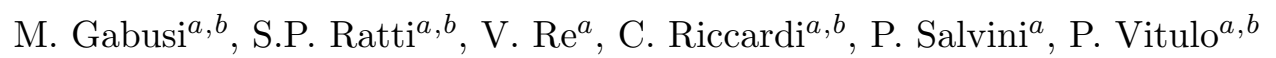

INFN Sezione di Perugia ${ }^{a}$, Università di Perugia ${ }^{b}$, Perugia, Italy

M. Biasini ${ }^{a, b}$, G.M. Bilei ${ }^{a}$, D. Ciangottini ${ }^{a, b}, 2$, L. Fanò ${ }^{a, b}$, P. Lariccia $^{a, b}$, G. Mantovani $^{a, b}{ }^{,}$ M. Menichelli ${ }^{a}$, A. Saha ${ }^{a}$, A. Santocchia ${ }^{a, b}$, A. Spiezia ${ }^{a, b, 2}$

INFN Sezione di Pisa ${ }^{a}$, Università di Pisa ${ }^{b}$, Scuola Normale Superiore di Pisa ${ }^{c}$, Pisa, Italy

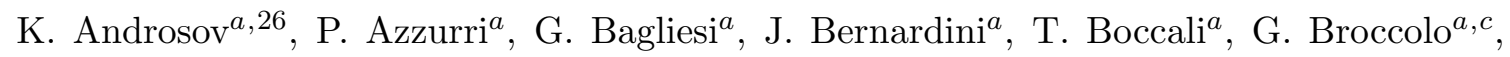
R. Castaldi ${ }^{a}$, M.A. Ciocci ${ }^{a, 26}$, R. Dell'Orso ${ }^{a}$, S. Donato ${ }^{a, c, 2}$, G. Fedi, F. Fiori ${ }^{a, c}$, L. Foà $^{a, c}$, A. Giassi ${ }^{a}$, M.T. Grippo ${ }^{a, 26}$, F. Ligabue ${ }^{a, c}$, T. Lomtadze ${ }^{a}$, L. Martini $^{a, b}$, A. Messineo $^{a, b}$,

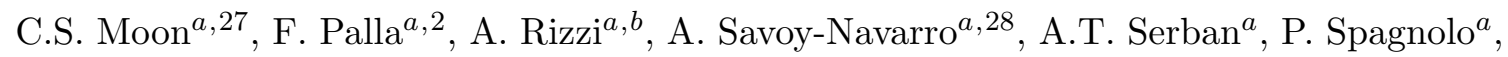

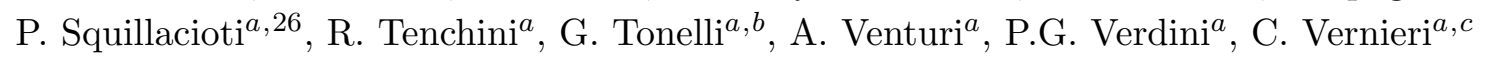

INFN Sezione di Roma ${ }^{a}$, Università di Roma ${ }^{b}$, Roma, Italy

L. Barone ${ }^{a, b}$, F. Cavallari ${ }^{a}$, G. D'imperio ${ }^{a, b}$, D. Del Re ${ }^{a, b}$, M. Diemoz ${ }^{a}$, C. Jorda ${ }^{a}$,

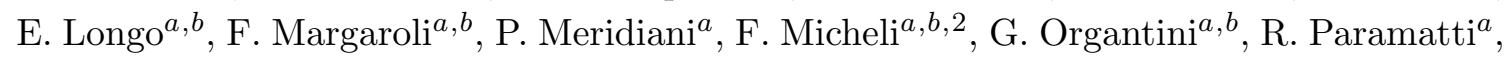

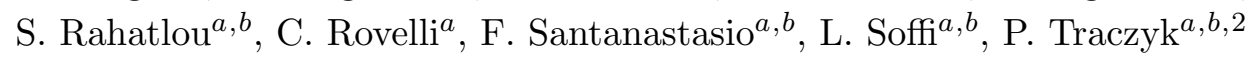

INFN Sezione di Torino ${ }^{a}$, Università di Torino ${ }^{b}$, Torino, Italy, Università del Piemonte Orientale ${ }^{c}$, Novara, Italy

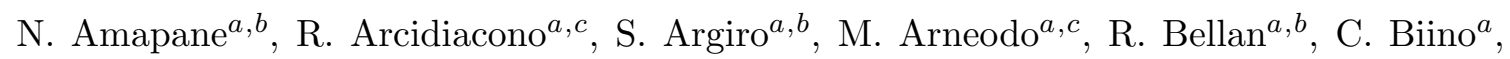
N. Cartiglia ${ }^{a}$, S. Casasso ${ }^{a, b, 2}$, M. Costa ${ }^{a, b}$, R. Covarelli, A. Degano ${ }^{a, b}$, N. Demaria ${ }^{a}$, L. Finco ${ }^{a, b, 2}$, C. Mariotti ${ }^{a}$, S. Maselli ${ }^{a}$, E. Migliore ${ }^{a, b}$, V. Monaco ${ }^{a, b}$, M. Musich ${ }^{a}$, M.M. Obertino ${ }^{a, c}$, L. Pacher ${ }^{a, b}$, N. Pastrone ${ }^{a}$, M. Pelliccioni ${ }^{a}$, G.L. Pinna Angioni ${ }^{a, b}$, A. Potenza ${ }^{a, b}$, A. Romero ${ }^{a, b}$, M. Ruspa ${ }^{a, c}$, R. $\operatorname{Sacchi}^{a, b}$, A. Solano ${ }^{a, b}$, A. Staiano ${ }^{a}$, U. Tamponi ${ }^{a}$

INFN Sezione di Trieste ${ }^{a}$, Università di Trieste ${ }^{b}$, Trieste, Italy

S. Belforte ${ }^{a}$, V. Candelise ${ }^{a, b, 2}$, M. Casarsa ${ }^{a}$, F. Cossutti ${ }^{a}$, G. Della Ricca ${ }^{a, b}$, B. Gobbo ${ }^{a}$,

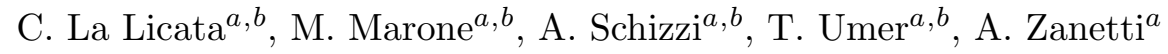


Kangwon National University, Chunchon, Korea

S. Chang, A. Kropivnitskaya, S.K. Nam

Kyungpook National University, Daegu, Korea

D.H. Kim, G.N. Kim, M.S. Kim, D.J. Kong, S. Lee, Y.D. Oh, H. Park, A. Sakharov, D.C. Son

Chonbuk National University, Jeonju, Korea

T.J. Kim, M.S. Ryu

Chonnam National University, Institute for Universe and Elementary Particles, Kwangju, Korea

J.Y. Kim, D.H. Moon, S. Song

Korea University, Seoul, Korea

S. Choi, D. Gyun, B. Hong, M. Jo, H. Kim, Y. Kim, B. Lee, K.S. Lee, S.K. Park, Y. Roh

Seoul National University, Seoul, Korea

H.D. Yoo

University of Seoul, Seoul, Korea

M. Choi, J.H. Kim, I.C. Park, G. Ryu

Sungkyunkwan University, Suwon, Korea

Y. Choi, Y.K. Choi, J. Goh, D. Kim, E. Kwon, J. Lee, I. Yu

Vilnius University, Vilnius, Lithuania

A. Juodagalvis

National Centre for Particle Physics, Universiti Malaya, Kuala Lumpur, Malaysia

J.R. Komaragiri, M.A.B. Md Ali ${ }^{29}$, W.A.T. Wan Abdullah

Centro de Investigacion y de Estudios Avanzados del IPN, Mexico City, Mexico

E. Casimiro Linares, H. Castilla-Valdez, E. De La Cruz-Burelo, I. Heredia-de La Cruz,

A. Hernandez-Almada, R. Lopez-Fernandez, A. Sanchez-Hernandez

Universidad Iberoamericana, Mexico City, Mexico

S. Carrillo Moreno, F. Vazquez Valencia

Benemerita Universidad Autonoma de Puebla, Puebla, Mexico

I. Pedraza, H.A. Salazar Ibarguen

Universidad Autónoma de San Luis Potosí, San Luis Potosí, Mexico

A. Morelos Pineda

University of Auckland, Auckland, New Zealand

D. Krofcheck

University of Canterbury, Christchurch, New Zealand

P.H. Butler, S. Reucroft 
National Centre for Physics, Quaid-I-Azam University, Islamabad, Pakistan

A. Ahmad, M. Ahmad, Q. Hassan, H.R. Hoorani, W.A. Khan, T. Khurshid, M. Shoaib

National Centre for Nuclear Research, Swierk, Poland

H. Bialkowska, M. Bluj, B. Boimska, T. Frueboes, M. Górski, M. Kazana, K. Nawrocki, K. Romanowska-Rybinska, M. Szleper, P. Zalewski

Institute of Experimental Physics, Faculty of Physics, University of Warsaw, Warsaw, Poland

G. Brona, K. Bunkowski, M. Cwiok, W. Dominik, K. Doroba, A. Kalinowski, M. Konecki, J. Krolikowski, M. Misiura, M. Olszewski

Laboratório de Instrumentação e Física Experimental de Partículas, Lisboa, Portugal

P. Bargassa, C. Beirão Da Cruz E Silva, P. Faccioli, P.G. Ferreira Parracho, M. Gallinaro,

L. Lloret Iglesias, F. Nguyen, J. Rodrigues Antunes, J. Seixas, D. Vadruccio, J. Varela, P. Vischia

Joint Institute for Nuclear Research, Dubna, Russia

P. Bunin, I. Golutvin, I. Gorbunov, V. Karjavin, V. Konoplyanikov, G. Kozlov, A. Lanev, A. Malakhov, V. Matveev ${ }^{30}$, P. Moisenz, V. Palichik, V. Perelygin, M. Savina, S. Shmatov,

S. Shulha, N. Skatchkov, V. Smirnov, A. Zarubin

Petersburg Nuclear Physics Institute, Gatchina (St. Petersburg), Russia

V. Golovtsov, Y. Ivanov, V. Kim ${ }^{31}$, E. Kuznetsova, P. Levchenko, V. Murzin, V. Oreshkin, I. Smirnov, V. Sulimov, L. Uvarov, S. Vavilov, A. Vorobyev, An. Vorobyev

Institute for Nuclear Research, Moscow, Russia

Yu. Andreev, A. Dermenev, S. Gninenko, N. Golubev, M. Kirsanov, N. Krasnikov, A. Pashenkov, D. Tlisov, A. Toropin

Institute for Theoretical and Experimental Physics, Moscow, Russia

V. Epshteyn, V. Gavrilov, N. Lychkovskaya, V. Popov, I. Pozdnyakov, G. Safronov, S. Semenov, A. Spiridonov, V. Stolin, E. Vlasov, A. Zhokin

P.N. Lebedev Physical Institute, Moscow, Russia

V. Andreev, M. Azarkin ${ }^{32}$, I. Dremin ${ }^{32}$, M. Kirakosyan, A. Leonidov ${ }^{32}$, G. Mesyats, S.V. Rusakov, A. Vinogradov

Skobeltsyn Institute of Nuclear Physics, Lomonosov Moscow State University, Moscow, Russia

A. Belyaev, E. Boos, M. Dubinin ${ }^{33}$, L. Dudko, A. Ershov, A. Gribushin, V. Klyukhin, O. Kodolova, I. Lokhtin, S. Obraztsov, S. Petrushanko, V. Savrin, A. Snigirev

State Research Center of Russian Federation, Institute for High Energy Physics, Protvino, Russia

I. Azhgirey, I. Bayshev, S. Bitioukov, V. Kachanov, A. Kalinin, D. Konstantinov,

V. Krychkine, V. Petrov, R. Ryutin, A. Sobol, L. Tourtchanovitch, S. Troshin, N. Tyurin, A. Uzunian, A. Volkov 
University of Belgrade, Faculty of Physics and Vinca Institute of Nuclear Sciences, Belgrade, Serbia

P. Adzic ${ }^{34}$, M. Ekmedzic, J. Milosevic, V. Rekovic

Centro de Investigaciones Energéticas Medioambientales y Tecnológicas (CIEMAT), Madrid, Spain

J. Alcaraz Maestre, C. Battilana, E. Calvo, M. Cerrada, M. Chamizo Llatas, N. Colino, B. De La Cruz, A. Delgado Peris, D. Domínguez Vázquez, A. Escalante Del Valle, C. Fernandez Bedoya, J.P. Fernández Ramos, J. Flix, M.C. Fouz, P. Garcia-Abia, O. Gonzalez Lopez, S. Goy Lopez, J.M. Hernandez, M.I. Josa, E. Navarro De Martino, A. Pérez-Calero Yzquierdo, J. Puerta Pelayo, A. Quintario Olmeda, I. Redondo, L. Romero, M.S. Soares

Universidad Autónoma de Madrid, Madrid, Spain

C. Albajar, J.F. de Trocóniz, M. Missiroli, D. Moran

\section{Universidad de Oviedo, Oviedo, Spain}

H. Brun, J. Cuevas, J. Fernandez Menendez, S. Folgueras, I. Gonzalez Caballero

Instituto de Física de Cantabria (IFCA), CSIC-Universidad de Cantabria, Santander, Spain

J.A. Brochero Cifuentes, I.J. Cabrillo, A. Calderon, J. Duarte Campderros, M. Fernandez, G. Gomez, A. Graziano, A. Lopez Virto, J. Marco, R. Marco, C. Martinez Rivero, F. Matorras, F.J. Munoz Sanchez, J. Piedra Gomez, T. Rodrigo, A.Y. Rodríguez-Marrero, A. Ruiz-Jimeno, L. Scodellaro, I. Vila, R. Vilar Cortabitarte

\section{CERN, European Organization for Nuclear Research, Geneva, Switzerland}

D. Abbaneo, E. Auffray, G. Auzinger, M. Bachtis, P. Baillon, A.H. Ball, D. Barney, A. Benaglia, J. Bendavid, L. Benhabib, J.F. Benitez, P. Bloch, A. Bocci, A. Bonato, O. Bondu, C. Botta, H. Breuker, T. Camporesi, G. Cerminara, S. Colafranceschi ${ }^{35}$, M. D'Alfonso, D. d'Enterria, A. Dabrowski, A. David, F. De Guio, A. De Roeck, S. De Visscher, E. Di Marco, M. Dobson, M. Dordevic, B. Dorney, N. Dupont-Sagorin, A. ElliottPeisert, G. Franzoni, W. Funk, D. Gigi, K. Gill, D. Giordano, M. Girone, F. Glege, R. Guida, S. Gundacker, M. Guthoff, J. Hammer, M. Hansen, P. Harris, J. Hegeman, V. Innocente, P. Janot, K. Kousouris, K. Krajczar, P. Lecoq, C. Lourenço, N. Magini, L. Malgeri, M. Mannelli, J. Marrouche, L. Masetti, F. Meijers, S. Mersi, E. Meschi, F. Moortgat, S. Morovic, M. Mulders, S. Orfanelli, L. Orsini, L. Pape, E. Perez, A. Petrilli, G. Petrucciani, A. Pfeiffer, M. Pimiä, D. Piparo, M. Plagge, A. Racz, G. Rolandi ${ }^{36}$, M. Rovere, H. Sakulin, C. Schäfer, C. Schwick, A. Sharma, P. Siegrist, P. Silva, M. Simon, P. Sphicas ${ }^{37}$, D. Spiga, J. Steggemann, B. Stieger, M. Stoye, Y. Takahashi, D. Treille, A. Tsirou, G.I. Veres ${ }^{18}$, N. Wardle, H.K. Wöhri, H. Wollny, W.D. Zeuner

\section{Paul Scherrer Institut, Villigen, Switzerland}

W. Bertl, K. Deiters, W. Erdmann, R. Horisberger, Q. Ingram, H.C. Kaestli, D. Kotlinski, U. Langenegger, D. Renker, T. Rohe 
Institute for Particle Physics, ETH Zurich, Zurich, Switzerland

F. Bachmair, L. Bäni, L. Bianchini, M.A. Buchmann, B. Casal, N. Chanon, G. Dissertori, M. Dittmar, M. Donegà, M. Dünser, P. Eller, C. Grab, D. Hits, J. Hoss, G. Kasieczka, W. Lustermann, B. Mangano, A.C. Marini, M. Marionneau, P. Martinez Ruiz del Arbol, M. Masciovecchio, D. Meister, N. Mohr, P. Musella, C. Nägeli ${ }^{38}$, F. Nessi-Tedaldi, F. Pandolfi, F. Pauss, L. Perrozzi, M. Peruzzi, M. Quittnat, L. Rebane, M. Rossini, A. Starodumov ${ }^{39}$, M. Takahashi, K. Theofilatos, R. Wallny, H.A. Weber

Universität Zürich, Zurich, Switzerland

C. Amsler ${ }^{40}$, M.F. Canelli, V. Chiochia, A. De Cosa, A. Hinzmann, T. Hreus, B. Kilminster, C. Lange, J. Ngadiuba, D. Pinna, P. Robmann, F.J. Ronga, S. Taroni, Y. Yang

National Central University, Chung-Li, Taiwan

M. Cardaci, K.H. Chen, C. Ferro, C.M. Kuo, W. Lin, Y.J. Lu, R. Volpe, S.S. Yu

National Taiwan University (NTU), Taipei, Taiwan

P. Chang, Y.H. Chang, Y. Chao, K.F. Chen, P.H. Chen, C. Dietz, U. Grundler, W.-S. Hou, Y.F. Liu, R.-S. Lu, M. Miñano Moya, E. Petrakou, J.F. Tsai, Y.M. Tzeng, R. Wilken

Chulalongkorn University, Faculty of Science, Department of Physics, Bangkok, Thailand

B. Asavapibhop, G. Singh, N. Srimanobhas, N. Suwonjandee

\section{Cukurova University, Adana, Turkey}

A. Adiguzel, M.N. Bakirci ${ }^{41}$, S. Cerci ${ }^{42}$, C. Dozen, I. Dumanoglu, E. Eskut, S. Girgis, G. Gokbulut, Y. Guler, E. Gurpinar, I. Hos, E.E. Kangal ${ }^{43}$, A. Kayis Topaksu, G. Onengut ${ }^{44}$, K. Ozdemir ${ }^{45}$, S. Ozturk ${ }^{41}$, A. Polatoz, D. Sunar Cerci ${ }^{42}$, B. Tali ${ }^{42}$, H. Topakli ${ }^{41}$, M. Vergili, C. Zorbilmez

Middle East Technical University, Physics Department, Ankara, Turkey

I.V. Akin, B. Bilin, S. Bilmis, H. Gamsizkan ${ }^{46}$, B. Isildak ${ }^{47}$, G. Karapinar ${ }^{48}$, K. Ocalan ${ }^{49}$, S. Sekmen, U.E. Surat, M. Yalvac, M. Zeyrek

Bogazici University, Istanbul, Turkey

E.A. Albayrak ${ }^{50}$, E. Gülmez, M. Kaya ${ }^{51}$, O. Kaya ${ }^{52}$, T. Yetkin ${ }^{53}$

Istanbul Technical University, Istanbul, Turkey

K. Cankocak, F.I. Vardarlı

National Scientific Center, Kharkov Institute of Physics and Technology, Kharkov, Ukraine

L. Levchuk, P. Sorokin

\section{University of Bristol, Bristol, United Kingdom}

J.J. Brooke, E. Clement, D. Cussans, H. Flacher, J. Goldstein, M. Grimes, G.P. Heath, H.F. Heath, J. Jacob, L. Kreczko, C. Lucas, Z. Meng, D.M. Newbold ${ }^{54}$, S. Paramesvaran, A. Poll, T. Sakuma, S. Seif El Nasr-storey, S. Senkin, V.J. Smith 


\section{Rutherford Appleton Laboratory, Didcot, United Kingdom}

K.W. Bell, A. Belyaev ${ }^{55}$, C. Brew, R.M. Brown, D.J.A. Cockerill, J.A. Coughlan, K. Harder, S. Harper, E. Olaiya, D. Petyt, C.H. Shepherd-Themistocleous, A. Thea, I.R. Tomalin, T. Williams, W.J. Womersley, S.D. Worm

\section{Imperial College, London, United Kingdom}

M. Baber, R. Bainbridge, O. Buchmuller, D. Burton, D. Colling, N. Cripps, P. Dauncey, G. Davies, M. Della Negra, P. Dunne, A. Elwood, W. Ferguson, J. Fulcher, D. Futyan, G. Hall, G. Iles, M. Jarvis, G. Karapostoli, M. Kenzie, R. Lane, R. Lucas ${ }^{54}$, L. Lyons, A.M. Magnan, S. Malik, B. Mathias, J. Nash, A. Nikitenko ${ }^{39}$, J. Pela, M. Pesaresi, K. Petridis, D.M. Raymond, S. Rogerson, A. Rose, C. Seez, P. Sharp ${ }^{\dagger}$, A. Tapper, M. Vazquez Acosta, T. Virdee, S.C. Zenz

\section{Brunel University, Uxbridge, United Kingdom}

J.E. Cole, P.R. Hobson, A. Khan, P. Kyberd, D. Leggat, D. Leslie, I.D. Reid, P. Symonds, L. Teodorescu, M. Turner

\section{Baylor University, Waco, USA}

J. Dittmann, K. Hatakeyama, A. Kasmi, H. Liu, N. Pastika, T. Scarborough, Z. Wu

The University of Alabama, Tuscaloosa, USA

O. Charaf, S.I. Cooper, C. Henderson, P. Rumerio

\section{Boston University, Boston, USA}

A. Avetisyan, T. Bose, C. Fantasia, P. Lawson, C. Richardson, J. Rohlf, J. St. John, L. Sulak

\section{Brown University, Providence, USA}

J. Alimena, E. Berry, S. Bhattacharya, G. Christopher, D. Cutts, Z. Demiragli, N. Dhingra, A. Ferapontov, A. Garabedian, U. Heintz, E. Laird, G. Landsberg, Z. Mao, M. Narain, S. Sagir, T. Sinthuprasith, T. Speer, J. Swanson

\section{University of California, Davis, Davis, USA}

R. Breedon, G. Breto, M. Calderon De La Barca Sanchez, S. Chauhan, M. Chertok, J. Conway, R. Conway, P.T. Cox, R. Erbacher, M. Gardner, W. Ko, R. Lander, M. Mulhearn, D. Pellett, J. Pilot, F. Ricci-Tam, S. Shalhout, J. Smith, M. Squires, D. Stolp, M. Tripathi, S. Wilbur, R. Yohay

\section{University of California, Los Angeles, USA}

R. Cousins, P. Everaerts, C. Farrell, J. Hauser, M. Ignatenko, G. Rakness, E. Takasugi, V. Valuev, M. Weber

\section{University of California, Riverside, Riverside, USA}

K. Burt, R. Clare, J. Ellison, J.W. Gary, G. Hanson, J. Heilman, M. Ivova Rikova, P. Jandir, E. Kennedy, F. Lacroix, O.R. Long, A. Luthra, M. Malberti, M. Olmedo Negrete, A. Shrinivas, S. Sumowidagdo, S. Wimpenny 


\section{University of California, San Diego, La Jolla, USA}

J.G. Branson, G.B. Cerati, S. Cittolin, R.T. D’Agnolo, A. Holzner, R. Kelley, D. Klein, J. Letts, I. Macneill, D. Olivito, S. Padhi, C. Palmer, M. Pieri, M. Sani, V. Sharma, S. Simon, M. Tadel, Y. Tu, A. Vartak, C. Welke, F. Würthwein, A. Yagil, G. Zevi Della Porta

\section{University of California, Santa Barbara, Santa Barbara, USA}

D. Barge, J. Bradmiller-Feld, C. Campagnari, T. Danielson, A. Dishaw, V. Dutta, K. Flowers, M. Franco Sevilla, P. Geffert, C. George, F. Golf, L. Gouskos, J. Incandela, C. Justus, N. Mccoll, S.D. Mullin, J. Richman, D. Stuart, W. To, C. West, J. Yoo

\section{California Institute of Technology, Pasadena, USA}

A. Apresyan, A. Bornheim, J. Bunn, Y. Chen, J. Duarte, A. Mott, H.B. Newman, C. Pena, M. Pierini, M. Spiropulu, J.R. Vlimant, R. Wilkinson, S. Xie, R.Y. Zhu

\section{Carnegie Mellon University, Pittsburgh, USA}

V. Azzolini, A. Calamba, B. Carlson, T. Ferguson, Y. Iiyama, M. Paulini, J. Russ, H. Vogel, I. Vorobiev

\section{University of Colorado at Boulder, Boulder, USA}

J.P. Cumalat, W.T. Ford, A. Gaz, M. Krohn, E. Luiggi Lopez, U. Nauenberg, J.G. Smith, K. Stenson, S.R. Wagner

\section{Cornell University, Ithaca, USA}

J. Alexander, A. Chatterjee, J. Chaves, J. Chu, S. Dittmer, N. Eggert, N. Mirman, G. Nicolas Kaufman, J.R. Patterson, A. Ryd, E. Salvati, L. Skinnari, W. Sun, W.D. Teo, J. Thom, J. Thompson, J. Tucker, Y. Weng, L. Winstrom, P. Wittich

\section{Fairfield University, Fairfield, USA}

D. Winn

\section{Fermi National Accelerator Laboratory, Batavia, USA}

S. Abdullin, M. Albrow, J. Anderson, G. Apollinari, L.A.T. Bauerdick, A. Beretvas, J. Berryhill, P.C. Bhat, G. Bolla, K. Burkett, J.N. Butler, H.W.K. Cheung, F. Chlebana, S. Cihangir, V.D. Elvira, I. Fisk, J. Freeman, E. Gottschalk, L. Gray, D. Green, S. Grünendahl, O. Gutsche, J. Hanlon, D. Hare, R.M. Harris, J. Hirschauer, B. Hooberman, S. Jindariani, M. Johnson, U. Joshi, B. Klima, B. Kreis, S. Kwan ${ }^{\dagger}$, J. Linacre, D. Lincoln, R. Lipton, T. Liu, R. Lopes De Sá, J. Lykken, K. Maeshima, J.M. Marraffino, V.I. Martinez Outschoorn, S. Maruyama, D. Mason, P. McBride, P. Merkel, K. Mishra, S. Mrenna, S. Nahn, C. Newman-Holmes, V. O’Dell, O. Prokofyev, E. Sexton-Kennedy, A. Soha, W.J. Spalding, L. Spiegel, L. Taylor, S. Tkaczyk, N.V. Tran, L. Uplegger, E.W. Vaandering, R. Vidal, A. Whitbeck, J. Whitmore, F. Yang

\section{University of Florida, Gainesville, USA}

D. Acosta, P. Avery, P. Bortignon, D. Bourilkov, M. Carver, D. Curry, S. Das, M. De Gruttola, G.P. Di Giovanni, R.D. Field, M. Fisher, I.K. Furic, J. Hugon, J. Konigsberg, A. Korytov, T. Kypreos, J.F. Low, K. Matchev, H. Mei, P. Milenovic ${ }^{56}$, G. Mitselmakher, L. Muniz, A. Rinkevicius, L. Shchutska, M. Snowball, D. Sperka, J. Yelton, M. Zakaria 
Florida International University, Miami, USA

S. Hewamanage, S. Linn, P. Markowitz, G. Martinez, J.L. Rodriguez

Florida State University, Tallahassee, USA

J.R. Adams, T. Adams, A. Askew, J. Bochenek, B. Diamond, J. Haas, S. Hagopian, V. Hagopian, K.F. Johnson, H. Prosper, V. Veeraraghavan, M. Weinberg

\section{Florida Institute of Technology, Melbourne, USA}

M.M. Baarmand, M. Hohlmann, H. Kalakhety, F. Yumiceva

University of Illinois at Chicago (UIC), Chicago, USA

M.R. Adams, L. Apanasevich, D. Berry, R.R. Betts, I. Bucinskaite, R. Cavanaugh, O. Evdokimov, L. Gauthier, C.E. Gerber, D.J. Hofman, P. Kurt, C. O'Brien, I.D. Sandoval Gonzalez, C. Silkworth, P. Turner, N. Varelas

The University of Iowa, Iowa City, USA

B. Bilki ${ }^{57}$, W. Clarida, K. Dilsiz, M. Haytmyradov, V. Khristenko, J.-P. Merlo, H. Mermerkaya ${ }^{58}$, A. Mestvirishvili, A. Moeller, J. Nachtman, H. Ogul, Y. Onel, F. Ozok ${ }^{50}$, A. Penzo, R. Rahmat, S. Sen, P. Tan, E. Tiras, J. Wetzel, K. Yi

Johns Hopkins University, Baltimore, USA

I. Anderson, B.A. Barnett, B. Blumenfeld, S. Bolognesi, D. Fehling, A.V. Gritsan, P. Maksimovic, C. Martin, M. Swartz, M. Xiao

The University of Kansas, Lawrence, USA

P. Baringer, A. Bean, G. Benelli, C. Bruner, J. Gray, R.P. Kenny III, D. Majumder, M. Malek, M. Murray, D. Noonan, S. Sanders, J. Sekaric, R. Stringer, Q. Wang, J.S. Wood

Kansas State University, Manhattan, USA

I. Chakaberia, A. Ivanov, K. Kaadze, S. Khalil, M. Makouski, Y. Maravin, L.K. Saini, N. Skhirtladze, I. Svintradze

Lawrence Livermore National Laboratory, Livermore, USA

J. Gronberg, D. Lange, F. Rebassoo, D. Wright

University of Maryland, College Park, USA

C. Anelli, A. Baden, A. Belloni, B. Calvert, S.C. Eno, J.A. Gomez, N.J. Hadley, S. Jabeen, R.G. Kellogg, T. Kolberg, Y. Lu, A.C. Mignerey, K. Pedro, Y.H. Shin, A. Skuja, M.B. Tonjes, S.C. Tonwar

\section{Massachusetts Institute of Technology, Cambridge, USA}

A. Apyan, R. Barbieri, K. Bierwagen, W. Busza, I.A. Cali, L. Di Matteo, G. Gomez Ceballos, M. Goncharov, D. Gulhan, M. Klute, Y.S. Lai, Y.-J. Lee, A. Levin, P.D. Luckey, C. Paus, D. Ralph, C. Roland, G. Roland, G.S.F. Stephans, K. Sumorok, D. Velicanu, J. Veverka, B. Wyslouch, M. Yang, M. Zanetti, V. Zhukova

University of Minnesota, Minneapolis, USA

B. Dahmes, A. Gude, S.C. Kao, K. Klapoetke, Y. Kubota, J. Mans, S. Nourbakhsh, R. Rusack, A. Singovsky, N. Tambe, J. Turkewitz 


\section{University of Mississippi, Oxford, USA}

J.G. Acosta, S. Oliveros

University of Nebraska-Lincoln, Lincoln, USA

E. Avdeeva, K. Bloom, S. Bose, D.R. Claes, A. Dominguez, R. Gonzalez Suarez, J. Keller,

D. Knowlton, I. Kravchenko, J. Lazo-Flores, F. Meier, F. Ratnikov, G.R. Snow, M. Zvada

State University of New York at Buffalo, Buffalo, USA

J. Dolen, A. Godshalk, I. Iashvili, A. Kharchilava, A. Kumar, S. Rappoccio

\section{Northeastern University, Boston, USA}

G. Alverson, E. Barberis, D. Baumgartel, M. Chasco, A. Massironi, D.M. Morse, D. Nash,

T. Orimoto, D. Trocino, R.-J. Wang, D. Wood, J. Zhang

Northwestern University, Evanston, USA

K.A. Hahn, A. Kubik, N. Mucia, N. Odell, B. Pollack, A. Pozdnyakov, M. Schmitt, S. Stoynev, K. Sung, M. Trovato, M. Velasco, S. Won

\section{University of Notre Dame, Notre Dame, USA}

A. Brinkerhoff, K.M. Chan, A. Drozdetskiy, M. Hildreth, C. Jessop, D.J. Karmgard, N. Kellams, K. Lannon, S. Lynch, N. Marinelli, Y. Musienko ${ }^{30}$, T. Pearson, M. Planer, R. Ruchti, G. Smith, N. Valls, M. Wayne, M. Wolf, A. Woodard

The Ohio State University, Columbus, USA

L. Antonelli, J. Brinson, B. Bylsma, L.S. Durkin, S. Flowers, A. Hart, C. Hill, R. Hughes, K. Kotov, T.Y. Ling, W. Luo, D. Puigh, M. Rodenburg, B.L. Winer, H. Wolfe, H.W. Wulsin

Princeton University, Princeton, USA

O. Driga, P. Elmer, J. Hardenbrook, P. Hebda, S.A. Koay, P. Lujan, D. Marlow, T. Medvedeva, M. Mooney, J. Olsen, P. Piroué, X. Quan, H. Saka, D. Stickland² , C. Tully, J.S. Werner, A. Zuranski

University of Puerto Rico, Mayaguez, USA

E. Brownson, S. Malik, H. Mendez, J.E. Ramirez Vargas

Purdue University, West Lafayette, USA

V.E. Barnes, D. Benedetti, D. Bortoletto, L. Gutay, Z. Hu, M.K. Jha, M. Jones, K. Jung, M. Kress, N. Leonardo, D.H. Miller, N. Neumeister, F. Primavera, B.C. Radburn-Smith, X. Shi, I. Shipsey, D. Silvers, A. Svyatkovskiy, F. Wang, W. Xie, L. Xu, J. Zablocki

Purdue University Calumet, Hammond, USA

N. Parashar, J. Stupak

\section{Rice University, Houston, USA}

A. Adair, B. Akgun, K.M. Ecklund, F.J.M. Geurts, W. Li, B. Michlin, B.P. Padley, R. Redjimi, J. Roberts, J. Zabel 
University of Rochester, Rochester, USA

B. Betchart, A. Bodek, P. de Barbaro, R. Demina, Y. Eshaq, T. Ferbel, M. Galanti, A. Garcia-Bellido, P. Goldenzweig, J. Han, A. Harel, O. Hindrichs, A. Khukhunaishvili, S. Korjenevski, G. Petrillo, M. Verzetti, D. Vishnevskiy

The Rockefeller University, New York, USA

R. Ciesielski, L. Demortier, K. Goulianos, C. Mesropian

Rutgers, The State University of New Jersey, Piscataway, USA

S. Arora, A. Barker, J.P. Chou, C. Contreras-Campana, E. Contreras-Campana, D. Duggan, D. Ferencek, Y. Gershtein, R. Gray, E. Halkiadakis, D. Hidas, E. Hughes, S. Kaplan, A. Lath, S. Panwalkar, M. Park, S. Salur, S. Schnetzer, D. Sheffield, S. Somalwar, R. Stone, S. Thomas, P. Thomassen, M. Walker

University of Tennessee, Knoxville, USA

K. Rose, S. Spanier, A. York

\section{Texas A\&M University, College Station, USA}

O. Bouhali ${ }^{59}$, A. Castaneda Hernandez, M. Dalchenko, M. De Mattia, S. Dildick, R. Eusebi, W. Flanagan, J. Gilmore, T. Kamon ${ }^{60}$, V. Khotilovich, V. Krutelyov, R. Montalvo, I. Osipenkov, Y. Pakhotin, R. Patel, A. Perloff, J. Roe, A. Rose, A. Safonov, I. Suarez, A. Tatarinov, K.A. Ulmer

Texas Tech University, Lubbock, USA

N. Akchurin, C. Cowden, J. Damgov, C. Dragoiu, P.R. Dudero, J. Faulkner, K. Kovitanggoon, S. Kunori, S.W. Lee, T. Libeiro, I. Volobouev

Vanderbilt University, Nashville, USA

E. Appelt, A.G. Delannoy, S. Greene, A. Gurrola, W. Johns, C. Maguire, Y. Mao, A. Melo, M. Sharma, P. Sheldon, B. Snook, S. Tuo, J. Velkovska

University of Virginia, Charlottesville, USA

M.W. Arenton, S. Boutle, B. Cox, B. Francis, J. Goodell, R. Hirosky, A. Ledovskoy, H. Li, C. Lin, C. Neu, E. Wolfe, J. Wood

Wayne State University, Detroit, USA

C. Clarke, R. Harr, P.E. Karchin, C. Kottachchi Kankanamge Don, P. Lamichhane, J. Sturdy

\section{University of Wisconsin, Madison, USA}

D.A. Belknap, D. Carlsmith, M. Cepeda, S. Dasu, L. Dodd, S. Duric, E. Friis, R. HallWilton, M. Herndon, A. Hervé, P. Klabbers, A. Lanaro, C. Lazaridis, A. Levine, R. Loveless, A. Mohapatra, I. Ojalvo, T. Perry, G.A. Pierro, G. Polese, I. Ross, T. Sarangi, A. Savin, W.H. Smith, D. Taylor, C. Vuosalo, N. Woods

$\dagger$ : Deceased 
1: Also at Vienna University of Technology, Vienna, Austria

2: Also at CERN, European Organization for Nuclear Research, Geneva, Switzerland

3: Also at Institut Pluridisciplinaire Hubert Curien, Université de Strasbourg, Université de Haute Alsace Mulhouse, CNRS/IN2P3, Strasbourg, France

4: Also at National Institute of Chemical Physics and Biophysics, Tallinn, Estonia

5: Also at Skobeltsyn Institute of Nuclear Physics, Lomonosov Moscow State University, Moscow, Russia

6: Also at Universidade Estadual de Campinas, Campinas, Brazil

7: Also at Laboratoire Leprince-Ringuet, Ecole Polytechnique, IN2P3-CNRS, Palaiseau, France

8: Also at Université Libre de Bruxelles, Bruxelles, Belgium

9: Also at Joint Institute for Nuclear Research, Dubna, Russia

10: Also at Suez University, Suez, Egypt

11: Also at Cairo University, Cairo, Egypt

12: Also at Fayoum University, El-Fayoum, Egypt

13: Also at British University in Egypt, Cairo, Egypt

14: Now at Ain Shams University, Cairo, Egypt

15: Also at Université de Haute Alsace, Mulhouse, France

16: Also at Brandenburg University of Technology, Cottbus, Germany

17: Also at Institute of Nuclear Research ATOMKI, Debrecen, Hungary

18: Also at Eötvös Loránd University, Budapest, Hungary

19: Also at University of Debrecen, Debrecen, Hungary

20: Also at University of Visva-Bharati, Santiniketan, India

21: Now at King Abdulaziz University, Jeddah, Saudi Arabia

22: Also at University of Ruhuna, Matara, Sri Lanka

23: Also at Isfahan University of Technology, Isfahan, Iran

24: Also at University of Tehran, Department of Engineering Science, Tehran, Iran

25: Also at Plasma Physics Research Center, Science and Research Branch, Islamic Azad University, Tehran, Iran

26: Also at Università degli Studi di Siena, Siena, Italy

27: Also at Centre National de la Recherche Scientifique (CNRS) - IN2P3, Paris, France

28: Also at Purdue University, West Lafayette, USA

29: Also at International Islamic University of Malaysia, Kuala Lumpur, Malaysia

30: Also at Institute for Nuclear Research, Moscow, Russia

31: Also at St. Petersburg State Polytechnical University, St. Petersburg, Russia

32: Also at National Research Nuclear University 'Moscow Engineering Physics Institute' (MEPhI), Moscow, Russia

33: Also at California Institute of Technology, Pasadena, USA

34: Also at Faculty of Physics, University of Belgrade, Belgrade, Serbia

35: Also at Facoltà Ingegneria, Università di Roma, Roma, Italy

36: Also at Scuola Normale e Sezione dell'INFN, Pisa, Italy

37: Also at University of Athens, Athens, Greece

38: Also at Paul Scherrer Institut, Villigen, Switzerland

39: Also at Institute for Theoretical and Experimental Physics, Moscow, Russia

40: Also at Albert Einstein Center for Fundamental Physics, Bern, Switzerland

41: Also at Gaziosmanpasa University, Tokat, Turkey

42: Also at Adiyaman University, Adiyaman, Turkey

43: Also at Mersin University, Mersin, Turkey

44: Also at Cag University, Mersin, Turkey 
45: Also at Piri Reis University, Istanbul, Turkey

46: Also at Anadolu University, Eskisehir, Turkey

47: Also at Ozyegin University, Istanbul, Turkey

48: Also at Izmir Institute of Technology, Izmir, Turkey

49: Also at Necmettin Erbakan University, Konya, Turkey

50: Also at Mimar Sinan University, Istanbul, Istanbul, Turkey

51: Also at Marmara University, Istanbul, Turkey

52: Also at Kafkas University, Kars, Turkey

53: Also at Yildiz Technical University, Istanbul, Turkey

54: Also at Rutherford Appleton Laboratory, Didcot, United Kingdom

55: Also at School of Physics and Astronomy, University of Southampton, Southampton, United Kingdom

56: Also at University of Belgrade, Faculty of Physics and Vinca Institute of Nuclear Sciences, Belgrade, Serbia

57: Also at Argonne National Laboratory, Argonne, USA

58: Also at Erzincan University, Erzincan, Turkey

59: Also at Texas A\&M University at Qatar, Doha, Qatar

60: Also at Kyungpook National University, Daegu, Korea 